In "Computational Intelligence in Digital Forensics: Forensic Investigation and Application", A. Muda, Y-H Choo, A. Abraham and S. N. Srihari (eds.), Springer 2014, pages 333-383.

\title{
Computational Methods for the Analysis of Footwear Impression Evidence $^{\star}$
}

\author{
Sargur N. Srihari and Yi Tang \\ University at Buffalo, The State University of New York \\ srihari@buffalo.edu
}

\begin{abstract}
Impressions of footwear are commonly found in crime scenes. Yet they are not routinely used as evidence due to: (i) the wide variability and quality of impressions, and (ii) the large number of footwear outsole designs which makes their manual comparison time-consuming and difficult. Computational methods hold the promise of better use of footwear evidence in investigations and also in providing assistance for court testimony. This paper begins with a comprehensive survey of existing methods, followed by identifying several gaps in technology. They include methods to improve image quality, computing features for comparison, measuring the degree of similarity, retrieval of closest prints from a database and determining the degree of uncertainty in identification. New algorithms for each of these problems are proposed. An end-to-end system is proposed where : (i) the print is represented by an attribute relational graph of straight edges and ellipses, (ii) a distance measure based on the earth-mover distance, (iii) clustering to speed-up database retrieval, and (iv) uncertainty evaluation based on likelihoods. Retrieval performance of the proposed design with real crime scene images is evaluated and compared to that of previous methods. Suggestions for further work and implications to the justice system are given.
\end{abstract}

Keywords Footwear, Impression evidence, Computational forensics, Image similarity, Crime scene images

\section{Introduction}

Marks made on floors, carpets and other surfaces by the sole of footwear, known as footwear impressions, are the most commonly found type of evidence in crime scenes. Outside soles of footwear, known as outsoles, contain patterns that are designed by the footwear manufacturer. The design is both for functionality, i.e., gripping the walking surface, and for aesthetics, i.e., pleasing appearance. The patterns can change in distinctive ways over time depending on length of wear and walk characteristics such as gait and pressure. Impressions are created when footwear is pressed or stamped against a surface such as floor or furniture, in which process, the characteristics of the outsole are transferred to the surface. Impressions can contain three-dimensional information, e.g., on snow, wet dirt or at the beach, but more often contain only twodimensional patterns, e.g., on a floor or carpet. They are said to be present more often and found more frequently than fingerprints. Evidence provided by a positively identified mark can be as strong as evidence provided by other types of impression evidence such as fingerprints, tool marks, and typewritten impressions [1].

Footwear impression patterns can be useful for either identifying the sole type or the individual sole that made the impression. If the mark is identified as having been made by an individual outsole, to the the exclusion of all others, then it is referred to as individualization. It is based on individualizing characteristics, which are random marks the sole has acquired during its life. Individualizing characteristics are unique to the particular footwear that is the source of the impression. They are attributable to shoe sole defects such as nicks, scratches, cuts, punctures, tears, embedded air bubbles caused by manufacturing imperfections, and ragged holes [2]. A combination of position, configuration, and orientation of each defect, which are the result of events that occurred in its life, are unique to each shoe. A defect position is characterized relative to: print perimeter, particular tread elements or portions of patterns, or other defects. A defect shape is

\footnotetext{
* This work was supported by the Office of Justice Programs, US Department of Justice on NIJ Award 2007-DNBX-K135. The opinions expressed are those of the authors and not of the DoJ.
} 
characterized by its length, width, and other shape measures. The rotational orientation of the defect helps differentiate from other similarly shaped defects.

A broader type of identification is classification to determine the specific sole type, e.g., brand, based on class characteristics, which are features of the sole type. Detail retained may be insufficient to uniquely identify an individual shoe but is still very valuable. Since a wide variety of footwear is available on the market, with most having distinctive outsole patterns, any specific model will be owned by a very small fraction of the general population, although the same outsole pattern can be found on several different footwear brands and models. If the outsole pattern can be determined from its mark, then this can significantly narrow the search for a particular suspect. Class characteristics are useful for discriminating between different sole types. They capture the geometry of the pattern. Since there are a large number of sole types, they can be used to narrow-down the possibilities. Determining sole type can be regarded as a problem of image retrieval where the query is the print of unknown type and the database consists of all known prints whose impressions can be obtained using a chemical surface. Individualizing and class characteristics together enable determining whether a crime scene print matches a known.

Although ubiquitous, the poor quality and wide variability of impressions as well as the large number of manufactured outsole patterns makes their analysis and courtroom presentation difficult. Even in Europe, where footwear impression evidence is more commonly used, it is not used as frequently as it could be. For example, only 500 of 14,000 recovered prints in the Netherlands were identified [3]. This is because footwear impressions are usually highly degraded, prints are inherently complex and databases are too large for manual comparison. There is variability in the quality of footwear impressions because of the variety of surfaces on which the impressions are made.

The rest of the paper is organized as follows. After a review of current practice in the US (Section 2), and the published computational literature (Section 3), algorithms for several subproblems are discussed in Section 4: image processing to improve image quality, extraction of features for class characterization, methods for measuring the similarity of prints, computing features for individualization, and quantifying opinion. Implications of the methods to practice as well as future work that needs to be done are indicated in Section 5.

\section{Current Practice}

The forensic examiner collects and preserves footwear and tire tread impression evidence, makes examinations, comparisons, and analyses in order to: (i) include, identify, or eliminate a particular footwear, or type of outsole, as the source of an impression, (ii) determine the brand or manufacturer of the outsole or footwear, (iii) link scenes of crime, and (iii) write reports and provide testimony as needed. The photograph of the impression or of the lifted impression or cast can be subsequently scanned and a digital image produced. Forensic analysis requires comparison of this image against other images such as: (i) marks made by footwear currently and previously available on the market and (ii) marks found at other crime scenes.

An image of a footwear impression can be obtained using photography, gel, or electrostatic lifting or by making a cast when the impression is in soil. Subsequently, in the forensic laboratory, the image is compared with prints and impressions of known footwear. In computerized identification, known prints (collected with care to capture of all possible impression information) are scanned, processed and indexed into a database, with the objective of retrieving the most likely matching prints.

Difficulty in identification is due to poor quality images and differences in environment between impression and knowns. While digital image enhancement, e.g., contextual thresholding, can enhance impression quality, debris, shadows and artifacts are difficult to filter out. Thus it is useful to segment the image into useable (impressed by footwear) and discardable regions (impressed by other artifacts such as debris).

European research has focused on tasks with important practical differences from the needs of US examiners. Impressions from scenes, assembled from several locations, are searched to find matches with crime scene impressions. Usable impressions are present in $30 \%$ of all burglaries [4], e.g., a study of several jurisdictions in Switzerland revealed that 35\% of crime scenes had usable footwear impressions, and $30 \%$ of all burglaries provide usable impressions[5]. Timely identification allows linking of crime scenes- since most crimes are committed by repeat offenders, several offenses are common in the same day, and offenders rarely discard 
footwear between crimes[6]. Since manual identification is laborious there exists a real need for automated methods.

Most crimes investigated in the US are homicides and assaults, not burglaries. In such cases, particularly homicides, it in unlikely that the same impression will appear in another case. Here the classification task of determining brand, style, size, gender etc., is of importance. Through classification, even if the person could not be identified, the search could be narrowed down to a smaller set of suspects. Forensic examiners of footwear and tire impression evidence are a community of about 200 professionals in the U. S. Guidelines for the profession are given by the Scientific Working Group on Footwear and Tire Tread Evidence (SWGTREAD). Footwear prints constitute about 80-90\% of the case-work of the tread examiner who deals with both footwear and tire-marks.

The final step in footwear impression evidence analysis is to state the result of comparison for presenting forensic evidence in the courtroom. In order to establish a uniform ground for interpreting footwear impression evidence, the ENFSI (European Network of Forensic Institutes) footwear impression and tool mark working group has proposed a 5-point conclusion scale ranging from identification, very strong (strong) support, moderately strong support, limited support.

\section{Existing Software and Algorithms}

Several software tools for processing and comparison of footwear impressions have been described in the literature. We provide here a summary of such methods as a backdrop for the algorithms we propose in Section 4. The earliest were semi-automatic methods of manually annotated footwear print descriptions using a codebook of shape primitives, e.g., wavy patterns, geometric shapes and logos $[7,8]$. The query print is also encoded in a similar manner. The most popular such systems today are SOLEMATE and SICAR $[9,10]$. These systems rely on manually encoding shoe-prints using a codebook of shapes and geometric primitives, such as wavy patterns, zig-zags, circles, triangles, and the query footwear impression requires it to be encoded in a similar manner. The process is laborious, time-consuming and can be the source of poor performance as the same pattern can be encoded differently by different users.

Although automatic classification of footwear prints is not yet practical, there are several published methods. A summary of the published retrieval methods and their performance is given in Table 1. Cumulative match score (CMS) is defined as follows. The identification process assumes a closed test, i.e., the true match is in the database. The input is compared to each entry in the database and the similarity scores are numerically ranked in descending order. If any of the top $r=1,5,10$ similarity scores corresponds to the input it is considered as a correct match. The percentage of times one of those $r$ similarity scores is the correct match for all inputs is referred to as the CMS.

In early work, Mikkonen and Astikainen (1994) [20] proposed a classification system in which codes based on basic shapes are used as a pattern descriptor. Geradts and Keijzer (1996) [3] described an automatic classification for outsole designs using Fourier features. The approach employs shapes generated from footwear prints using image morphology operators. Spatial positioning and frequencies of shapes are used for classification with a neural network. No performance measures are reported. Alexander et al. (1999) [4] presented a fractal pattern matching technique with mean square noise error as a matching criteria to match the collected impression against database prints.

Fourier descriptors, which are invariant to translation and rotation, have also been used for classification of full and partial prints [21,11]. First and fifth rank classification are $65 \%$ and $87 \%$ on full-prints, and $55 \%$ and $78 \%$ for partials. The approach shows that although footwear prints are processed globally they are encoded in terms of the local information evident in the print. In [12] pattern edge information is employed for classification. After image de-noising and smoothing operations, extracted edge directions are grouped into a quantized set of 72 bins at five degree intervals. This generates an edge direction histogram for each pattern which after applying a Discrete FT provides a description with scale, translational and rotational invariance. The approach deals well with variations, however query examples originate from the learning set and no performance is given for partial prints.

de Chazal et al. (2005) [11] proposed a fully automated shoe print classification system which uses power spectral density (PSD) of the print as a pattern descriptor. Here, PSD is invariant to translation and 
Table 1. Survey of algorithms for automatic footwear print retrieval.

\begin{tabular}{|c|c|c|c|c|c|c|c|c|c|c|c|c|}
\hline \multirow[t]{3}{*}{ Authors } & \multirow[t]{3}{*}{ Features } & \multicolumn{9}{|c|}{ Performance (Cumulative Match Score) } & \multirow[t]{3}{*}{ Limitations } & \multirow[t]{3}{*}{ Dataset } \\
\hline & & \multicolumn{3}{|c|}{ Full Prints } & \multicolumn{3}{|c|}{ Partials } & \multicolumn{3}{|c|}{ Crime Scene } & & \\
\hline & & $\begin{array}{l}@ 1 \\
\%\end{array}$ & $\begin{array}{l}@ 5 \\
\%\end{array}$ & $\begin{array}{l}@ 10 \\
\%\end{array}$ & $\begin{array}{l}\text { @1 } \\
\%\end{array}$ & $\begin{array}{l}@ 5 \\
\%\end{array}$ & $\begin{array}{l}@ 10 \\
\%\end{array}$ & $\begin{array}{l}@ 1 \\
\%\end{array}$ & $\begin{array}{l}@ 5 \\
\%\end{array}$ & $\begin{array}{l}@ 10 \\
\%\end{array}$ & & \\
\hline $\begin{array}{l}\text { deChazal, } \\
\text { Flynn, } \\
\text { et.al.(2005) } \\
{[11]}\end{array}$ & $\mid \begin{array}{l}\text { Power spec- } \\
\text { tral density } \\
(\text { PSD) }\end{array}$ & 64 & 87 & 90 & $\mid 50$ & 70 & |77 & - & - & - & $\begin{array}{l}\text { No Scaling in- } \\
\text { variance }\end{array}$ & $\begin{array}{l}475 \text { prints from } \\
\text { For. Sci. Lab, } \\
\text { Ireland }\end{array}$ \\
\hline $\begin{array}{l}\text { Zhang, } \\
\text { Allinson } \\
(2005)[12]\end{array}$ & $\begin{array}{|lr|}\text { Edge } & \text { direc- } \\
\text { tion, } & \text { FT } \\
\text { histogram }\end{array}$ & 85 & 95 & 97 & - & - & - & - & - & - & No partials & 512 prints \\
\hline $\begin{array}{l}\text { Pavlou, } \\
\text { Allinson } \\
(2006)[13]\end{array}$ & SIFT & 86 & 90 & 93 & 85 & 90 & 92 & - & - & - & No SoCs & $\begin{array}{l}368 \text { prints of } \\
\text { Forensic } \quad \text { Sci. } \\
\text { Serv., UK }\end{array}$ \\
\hline $\begin{array}{l}\text { Crookes, } \\
\text { Bouridane, } \\
\text { Su, Gueham } \\
(2007)[14] \\
\end{array}$ & $\begin{array}{l}\text { Local Image } \\
\text { Features } \\
(\text { LIF })\end{array}$ & 100 & 100 & 100 & 100 & 100 & 100 & - & - & - & $\begin{array}{l}\text { Synthesized } \\
\text { SoCs }\end{array}$ & $\begin{array}{lr}500 & \text { clean } \\
\text { prints, } & 50 \\
\text { degraded } & \end{array}$ \\
\hline $\begin{array}{l}\text { Crookes, } \\
\text { Bouridane, } \\
\text { Su, Gueham } \\
(2007)[14]\end{array}$ & $\begin{array}{l}\text { Phase Only } \\
\text { Correlation } \\
(\text { POC) }\end{array}$ & 100 & 100 & 100 & 100 & 100 & 100 & - & - & - & $\begin{array}{l}\text { No rotational } \\
\text { invariance }\end{array}$ & $\begin{array}{lr}100 & \text { clean } \\
\text { prints, } & 64 \\
\text { synthetic } & \\
\end{array}$ \\
\hline $\begin{array}{l}\text { Gueham, } \\
\text { Bouridane, } \\
\text { Crookes } \\
(2008)[15] \\
\end{array}$ & $\mathrm{POC}$ & - & - & - & - & - & 96 & - & - & - & $\begin{array}{l}\text { Tested with } 200 \\
\text { prints }\end{array}$ & \\
\hline $\begin{array}{l}\text { Patil, } \\
\text { Kulkarni } \\
(2009)[16]\end{array}$ & $\begin{array}{l}\text { Gabor trans- } \\
\text { form }\end{array}$ & 100 & 100 & 100 & 100 & 100 & 100 & - & - & - & $\begin{array}{llr}\text { No } & \text { SoCs } & \text { (fea- } \\
\text { tures } & \text { rely on } \\
\text { pixel } & \text { intensi- } \\
\text { ties) } & \end{array}$ & $\begin{array}{l}1400 \text { clean } \\
\text { full/partial \& } \\
\text { some synthetic } \\
\text { noisy prints }\end{array}$ \\
\hline \begin{tabular}{|l|} 
Dardi, \\
Cervelli, \\
Carrato \\
$(2009)[17]$ \\
\end{tabular} & Texture & - & - & - & - & - & - & 10 & 40 & 73 & $\begin{array}{l}\text { Tested with } 87 \\
\text { known prints } \\
\text { and } 30 \text { SoCs }\end{array}$ & $\begin{array}{lcc}87 & \text { known } & \text { and } \\
30 & \text { real } & \text { SoC } \\
\text { ENSFI } & \end{array}$ \\
\hline $\begin{array}{l}\text { Tang, Srihari } \\
(2010)[18, \\
19]\end{array}$ & $\begin{array}{l}\text { Shape Att. } \\
\text { Relational } \\
\text { Graph } \\
\text { (ARG) }\end{array}$ & 100 & 100 & 100 & 100 & 100 & 100 & 70 & 90 & 92 & $\begin{array}{l}\text { Slow speed } \\
\text { (compensated } \\
\text { by clustering) }\end{array}$ & $\begin{array}{l}1400 \text { degraded, } \\
1000 \text { known \& } \\
50 \text { real SoC }\end{array}$ \\
\hline
\end{tabular}


rotation of an image, crucial information of the print is preserved by removing the low and high frequency components and 2D correlation coefficient is used as similarity measure. Zhang and Allinson (2005) [12] proposed an automated shoe print retrieval system in which edge direction histogram is used to represent the shapes in shoes. The features consist of 1-D discrete Fourier Transform (FT) on the normalized edge direction histogram and the Euclidean distance is used as similarity measure.

Feature-point based methods, such as SIFT (Scale invariant feature transform) [22], have demonstrated good performance in general content-based image retrieval due to invariance with respect to scale, rotation and translation. However, they may be inappropriate for footwear impressions. This is partly because, as local extrema in the scale space, SIFT key points may not be preserved both among different shoes of the same class and through the life-time of a shoe. This problem is further complicated by the extremely poor quality and incompleteness of crime scene footwear impressions. Pavlou and Allinson (2006) [13] presented classification results where maximally stable extremal region (MSER) feature detectors are encoded with SIFT descriptors as features after which a Gaussian feature similarity matrix and Gaussian proximity matrix are used as the similarity measure. In some crime scenes, only partial shoe-prints (termed as "half prints" and "quarter prints") are available. Partial matching has to focus on how to fully make use of regions available, with the accuracy of matching algorithms decreasing with print size.

Ghouti et al. (2006) [23] describe a so-called ShoeHash approach for classification where directional filter banks (DFB) are used to capture local/global details of shoe-prints with energy dominant blocks used as feature vector and normalized Euclidean-distance similarity. Su et al. (2007) [24] proposed a shoe-print retrieval system based on topological and pattern spectra, where a pattern spectrum is constructed using the area measure of granulometry, the topological spectrum constructed using the Euler number and a normalized hybrid measure of both used for matching. Crookes et al. (2007) [14] described two ways to classify shoe-prints: (i) in the spatial domain, modification of existing techniques: Harris-Laplace detectors and SIFT descriptors is proposed; the Harris corner detector is used to find local features; Laplace based automatic scale selection is used to decide the final local features and a nearest neighbor similarity measure, and (ii) in the transform domain, phase-only correlation (POC) is used to match shoe-prints. Gueham et al. (2008) [15] evaluated the performance of Optimum Trade-off Synthetic Discriminant Function (OTSDF) filter and unconstrained OTSDF filter in classifying partial shoe-prints.

As an exercise in data mining, Sun et. al. [25] clustered shoe outsoles using color (RGB) information as features where the number of clusters $k$ was varied from 2 to 7 and the clustering results of $k$-means and expectation maximization were compared; the results are of limited use since RGB information of outsole photographs are absent in impression evidence. Algarni and Hamiane (2009) [26] proposed a retrieval system using Hu's moment invariants as features and compared similarity measures: Euclidean, city block, Canberra and correlation distance. Xiao and Shi (2008) [27] presented matching using PSD and Zernike moments. Jing et al. (2009) [28] presented a new feature, directionality. Here, features extracted from co-occurrence matrix, Fourier transform and directional mask are matched using sum-of-absolute-difference. Nibouche et al. (2009) [29] proposed a solution for matching rotated partial prints. Harris points encoded with SIFT descriptors are used as features and they are matched using random sample consensus (RANSAC).

Dardi et al. (2009) [17] described a texture based retrieval system. A Mahalanobis map is used to capture texture and then matched using a correlation co-efficient measure. In subsequent work [30,31] they offer a cumulative match score comparison between Mahanalobis, [11] and [15]. Wang et al. (2009) [32] presented a wavelet and fuzzy neural network approach. Patil and Kulkarni (2009) [16] proposed using the Gabor transform to extract multi-resolution features and then Euclidean distance for matching. Rotation is estimated by the Radon transform and compensated by rotating in the opposite direction.

While footwear impression image analysis methods have been described in many papers, there are many gaps in the technology, e.g., among the many image processing and feature extraction algorithms it is not clear as to which ones are best suited for the task of retrieving reference images from a database (in response to a real crime scene query). More generally, methods are needed to: enhance image quality, represent patterns commonly found in footwear prints (that are also useful for comparison), determine the degree of similarity between evidence and known, retrieve closest matches in a reference data set, map comparison results to an opinion scale, and combine multiple scene images from the same source. 


\section{Proposed Methods and Algorithms}

We describe here methods and algorithms for the following tasks:

1. Data sets for the design and evaluation of algorithms (Section 4.1).

2. Enhancing the quality of crime scene images for further processing (Section 4.2)

3. Representing footwear outsole patterns by extracting: (i) class characteristics and (ii) individualizing characteristics (Sections 4.3-4.5).

4. Similarity measures between patterns for use in comparison, retrieval and individualization (Section 4.6).

5. Search algorithms, including performance metrics and clustering of reference patterns. (Section 4.7).

6. Characterizing uncertainty of match between evidence and known (Section 4.8).

\subsection{Data Sets}

The development and evaluation of algorithms for any pattern analysis and recognition task critically depends upon the availability of data sets. They are used for training machine learning algorithms and for testing their performance. The data sets should ideally be representative of the population since the methods themselves are based on statistical analysis of the data. Three types of commonly used footwear print data sets are: digital images of outsoles provided by manufacturers, simulated crime scene images, and real crime scene images. Examples of such data sets are given below.

Photographs of Outsoles. Footwear manufacturers usually make available images of outsoles and uppers on commercial websites. A web crawler can visit a given set of vendor websites. and recover such images. An example of the types of images available is given in Fig. 1. About 10,000 such images were downloaded for the purpose of design and evaluation of algorithms.

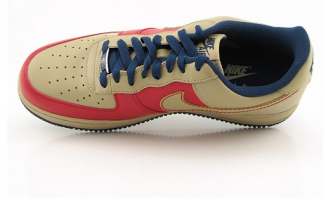

(a)

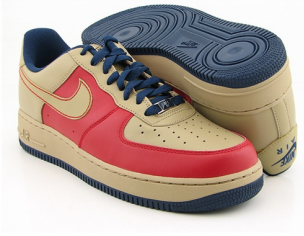

(b)

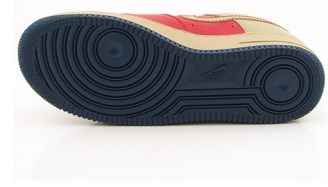

(c)

Fig. 1. Digital images of footwear outsoles and uppers available on the web. The particular model shown is called "Nike Air Force 1" which is most often encountered in U. S. crime scenes.

Simulated Scene Images. The process of recovery of prints in a crime scene is described in [1]. To create simulated crime scene prints, people are asked to step on powder and then onto a carpet to create a simulated crime scene print. Then the picture of the print is taken with a forensic scale near the print. The resolution of the images is calculated using the scale in the images and then scaled to $100 \mathrm{dpi}$. The prints are also captured on chemical paper to create the reference print. A chemical print is the known print which is obtained by a person stamping on a chemical pad and then on chemical paper, which would leave clear print on the paper. The chemical prints are converted into digital camera images of resolution 100dpi examples of which are shown in Fig. 2. Since the simulated crime scene prints tend to be of relatively high quality this leads to over-optimistic results in verification and identification. 


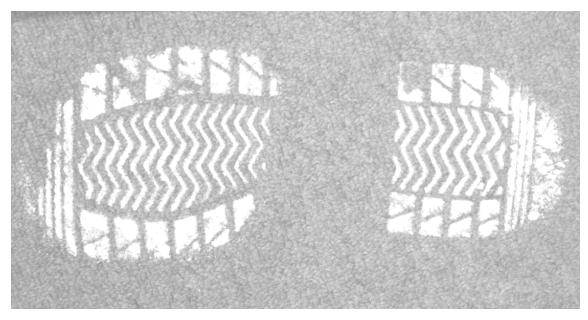

(a)

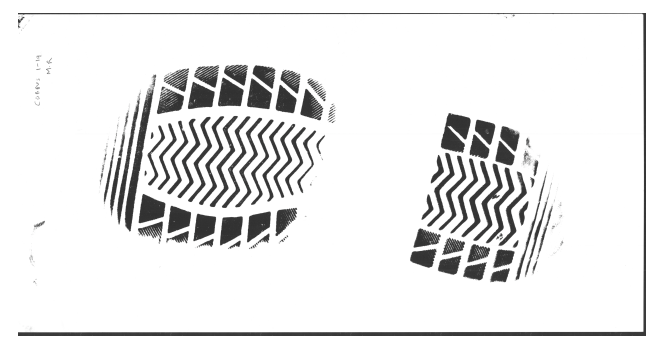

(b)

Fig. 2. Simulated crime scene and reference images: (a) print on carpet with powder, and (b) print on sheet of chemical paper.

Crime Scene and Reference Images. Statistical models are best constructed from actual crime scene images and the reference data sets used with them. However these are generally hard to find. Some examples from a database of 350 crime images are shown in Fig. 3 together with the ground-truth in Fig. 5. Reference prints can be obtained by taking impressions of footwear outsoles provided by footwear vendors-- some examples from a set of 5,000 reference prints are shown in Figure 4.

There are multiple prints from the same scene, e.g., in the first set 194 scene images are from 176 crime scenes and 144 scene images in the second are from 126 crimes. Each of the 50 scene images in the first dataset came from a different crime scene. Among these there are multiple shoe prints such as two partial shoe marks from the same crime scene, same marks taken at different illumination, same marks taken at different angles/orientation etc.

The resolution of reference images varies from 72 dpi to 150 dpi. Scene image resolution varies from 72 dpi to $240 \mathrm{dpi}$. The scene image dataset contains an equal number of color and gray-scale images. Only $3 \%$ of the reference images are direct photographs of the outsole of brand new shoes. The reference images can be broke down as follows. $97 \%$ are gray scale images. they are actually prints. $3 \%$ are color images, which are direct photographs of the outsole of the shoes on the market. Very few (less than $0.1 \%$ ) are binary images.

\subsection{Enhancing Image Quality}

The matching of crime scene impressions to known prints largely depends on the quality of the extracted image from the crime scene impression. Thus the first step in dealing with both crime scene prints and database prints is that of processing them in a way that makes further processing more effective and/or efficient. Two approaches are: image labeling and edge detection. In image labeling, different pixels or regions of the image are labeled as foreground (impression) or background; it can be done using either thresholding or pixel classification.

Thresholding. One simple method of labeling images as foreground/background is global thresholding. A threshold value for the gray-scale is selected and all pixels with an intensity lower than this value are marked as background and all pixels with higher values are marked as foreground. Different strategies for determining the global thresholding value exist. A simplistic method, for example, models the intensities as a histogram with the assumption of two main intensity peaks (foreground and background), selecting a middle point as the threshold. A more sophisticated method is Otsu thresholding [33] which is based on a threshold which minimizes weighted within class variance. Another method is a neural network, e.g., one with two layers with four input neurons, three middle layer neurons and one sigmoidal output. 


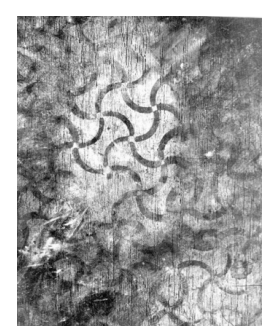

(a)

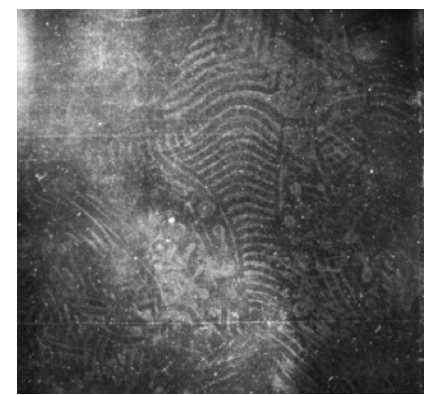

(b)

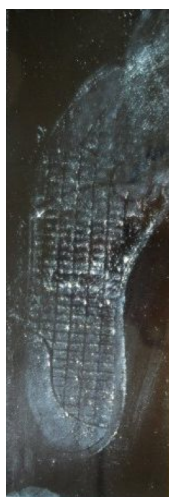

(c)

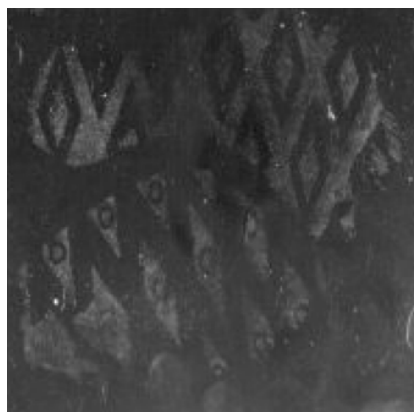

(d)

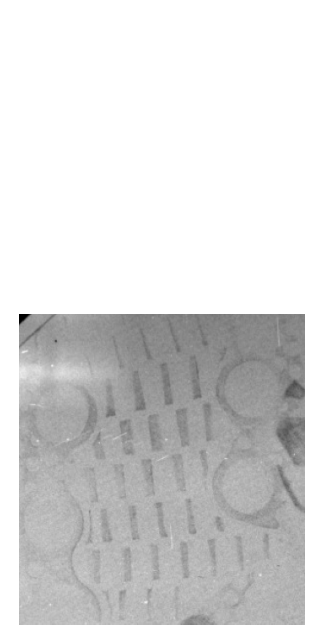

(e)

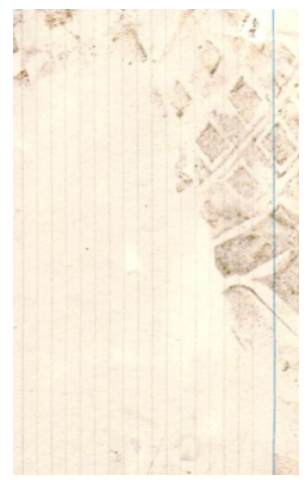

(f)

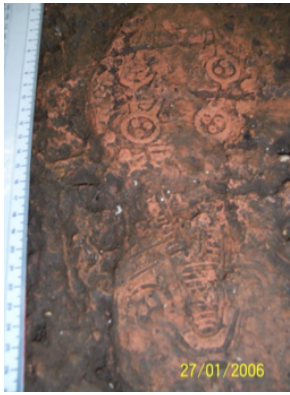

(g)

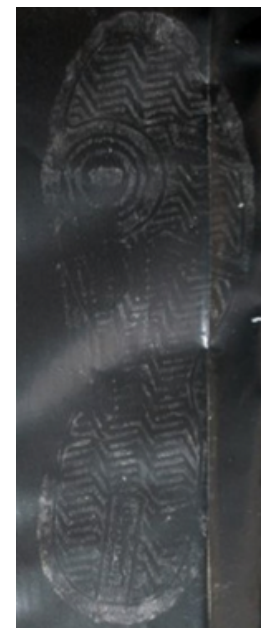

(h)

Fig. 3. Some crime scene images.

Adaptive Thresholding. A drawback of global thresholding is inability to cope with images that have a variety of intensities. An impression on carpet, for example, is often difficult to threshold since when the background is completely below/above the chosen threshold value, large portions of the print will also be missing. A solution is adaptive thresholding. Instead of selecting a single threshold value for the entire image, it is dynamically determined throughout the image. This can cope with larger changes in background, such as variations in background material (carpet, flooring, etc.) and lighting. Such images often lack the separation of peaks necessary to use global thresholding. Smaller sub-images are much more likely to be more uniform than the image overall. It selects the threshold value for each individual pixel based on the local neighborhood's range of pixel intensities. For some $n$ pixels around a given pixel, the thresholding value is calculated via mean, median, mean-C, etc. and used to determine whether a single pixel is part of the foreground or background, with different selections of sampling giving different results. After tuning the method to shoe-prints, this method gives high quality results at reasonable resolution. Some sample images are shown in Figure 6. 


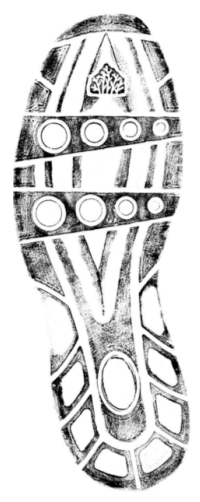

(a)

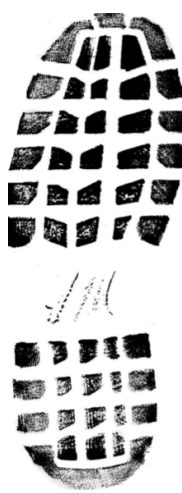

(b)

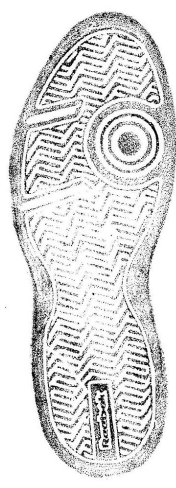

(h)

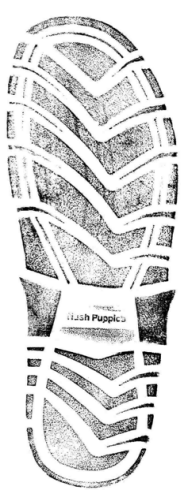

(c)

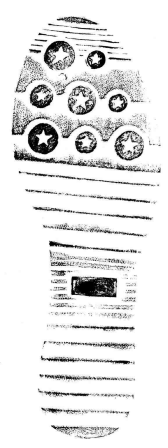

(i)

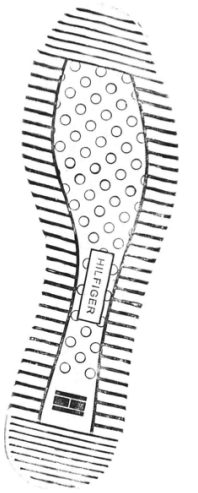

(d)

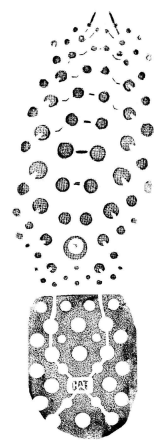

(j)

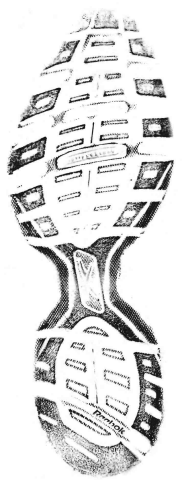

(e)

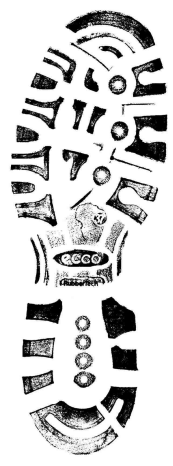

(k)

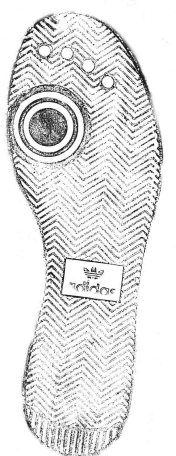

(f)

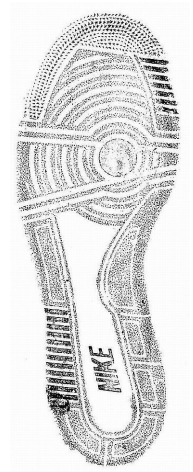

(1)

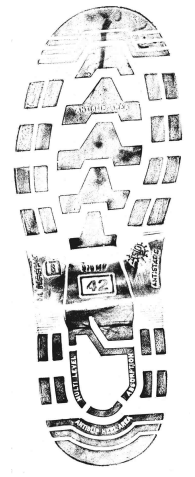

(g)

Fig. 4. Some reference images (knowns).

CRF Classification. While thresholding algorithms take into account only the value of the pixel or at most the surrounding pixels in making a decision, the information contained in other areas of the print can be useful. in inferring whether a pixel belongs to the foreground or background. The contextual information from other regions can be incorporated using probabilistic models known as conditional random fields(CRFs) [34]. CRFs are partially directed probabilistic graphical models [35] which belong to a class of machine learning models known as discriminative models, as opposed to generative models which need a full joint distribution to be estimated before a decision can be made. CRFs have been successfully used in image segmentation problems including documents containing handwriting [36]. The model exploits the inherent long range dependencies that exist in the images and hence is more robust than approaches using neural networks and other binarization algorithms. Here we describe the application of the CRF model to labelling pixels in footwear print images.

Our task is to learn a mapping from image $\mathbf{x}$ to labels $y$. Each $y$ is a member of a set of possible image labels $\mathcal{Y}=\{$ Impression, Background $\}$. The input image $\mathbf{x}$ is segmented into $m$ "patches" $\mathbf{x}=\left\{x_{1}, x_{2}, . ., x_{m}\right\}$. The patch size is chosen to be small enough for high resolution and big enough to extract enough features. We choose non-overlapping patches, $3 \times 3$ pixels. A CRF is used to label each patch using the labels of the neighboring patches.

The probabilistic CRF model is as follows. Using the Hamersley-Clifford theorem $p(y \mid \mathbf{x})=\frac{1}{Z} \prod_{i} \phi_{i}\left(D_{i}\right)$ where the $D_{i}$ are cliques of nodes in the graph with potentials $\phi_{i}$. Here node variables correspond to patches 


\begin{tabular}{|c|c|c|c|c|c|c|c|}
\hline 4 & & Answers for & imes and Matches (144 images from $126 \mathrm{cr}$ & imes Jan-De & ac'07]" Test D & & \\
\hline 3 & & & & & & & \\
\hline 2 & & & & & & & \\
\hline-1 & Test image No & Brand & Nodells) & imageid & Group Brand & Group Nodel(s). & Group imagelD \\
\hline (1) & (1) & & & & & & \\
\hline 1 & $\begin{array}{l}13101.2007011 \\
23101-2007-02\end{array}$ & KSwiss. & Finzier, Rinzier _eweil, Rinzier se. & 56907 & & & \\
\hline 3 & $\begin{array}{l}\text { 2:31:01/2007 } 02 \\
9174 \mathrm{jm2}\end{array}$ & Adidas & Thalcke & 22262 & & & \\
\hline 4 & EWPOC2001 & Lacoste & Solano, Koca & 53151 & & & \\
\hline 5 & Sosc conio & Untoro & Kingston & 62834 & & & \\
\hline 6 & $5: \operatorname{Dsc} 00431.1$ & Puma & Race Cat J, Rep Cat Msd J, Speed Cat & 15412 & & & \\
\hline & TAF & & Chorle $\cdots \cdots-\cdots-\cdots$ & 450 & Adiks & Beth, Wikox Yersa & 141 \\
\hline : & :FCOTPRIVT 35S.0I & Nike. & Ar Max Huaroche 2 & 42280 & & & \\
\hline : & 9:FCOTPRINT-355-C2 & & 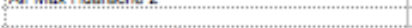 & 422000 & & & \\
\hline 10. & D.FOOTPRINT 361.01 & Nibe. & Enun Camas Blazer Suede. & 27636 & Pepe ceans & Action Leathe shoo. & 158277 \\
\hline 111 & 1.FCOTPRINT 361.02 & semen & 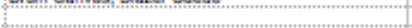 & trovo & Untoro & COO $100 \mathrm{IEP}$ & 7376 \\
\hline 12 & 2:FCOTPRINT 367 -01 & Ascot & 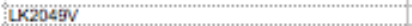 & 45662 & Kswss & Colyer Colpet & 54494 \\
\hline 13 & FOOTPRIVT 367.02 & & & & & & \\
\hline 14 & 4 FOOIPR NT 37000 & Adicos & उeo shei & 22065 & & & \\
\hline 10 & SCOTPRIVT $374.01 \mathrm{~W}$ & Giongo: & Logner boot. & 28847 & Howged Trail & Boot & 41203 \\
\hline 16 & FCOIPRIVT $374 . \mathrm{CL}$ & & & & Wrangles & orow & 28738 \\
\hline 17 & P:FCOTPRINT 374.03 & & & & & & \\
\hline 18 & 3: FOOTPRINT-394-1 & Vons & Lancet, Lani & 44596 & & & \\
\hline 19 & 9OOTPRINT 3942 & & & & & & \\
\hline 20 & FOOTPRINT $396-01$ & Nhe & Air Force 1, Air Force Low & 42251 & & & \\
\hline 21 & 1 FOOTPRANT-397-01 & Rectock & Classic Leather Mustang & 23422 & & & \\
\hline 2 & 2. FOOIPAINT $397=02$ & & & & ....... & & \\
\hline 要 & FOOTPANT $397-03$ & & & & & & \\
\hline 24 & 4 FOOTPRINT 398-01 & Elox & Blaze, Houston & 26825 & & & \\
\hline 28 & S:FOOTPRINT-401-01 & & & & Path & Yogl, Yog Low Skate & 31703 \\
\hline 26 & 5: FOOTPRINT $401=02$ & & & & & & \\
\hline 27 & 7 FOOTPRINT $401-03$ & & & & & & \\
\hline 28 & 9:FOOTPRINT-401-04 & & & & & & \\
\hline 29 & 9:FOOTPRINT-401-05 & & & & & & \\
\hline 30 & D: FOOTPRINT-402-01 & Lacoste & Score, Score V, Score Strap & 53147 & & & \\
\hline 31 & 1 FOOTPRINT $402-02$ & Nhe & Air Force I, Air Force Low & 42251 & Elesse & Eaham, Bologna Mid & 75317 \\
\hline 32 & 2: FOOTPAINT - $404-01$ & ugz & Hero, Gothic, sheild (Zroos) & segge & & & \\
\hline 33 & B FOOTPRINI - $404-02$ & & & & …..... & (1) & \\
\hline 34 & 4 FOOTPANTT-404-03 & & & & & & \\
\hline 36 & SFOOTPRNT $404-04$ & & & & & & \\
\hline 36 & 5: FOOTPRINT-405-01 & Grisport & Contractor, Directoc, Combat & 15205 & Atlan & Bolti, Boltimore & 30622 \\
\hline 37 & 7 FoOTPAUNT $407-01$ & Fó & ngleton & 51241 & $\mathrm{NX2}$ & 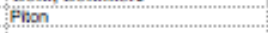 & 71727 \\
\hline 3is & FOOTPANTT $407=02$ & & & & & & \\
\hline 39 & FOOTPANT $407-03$ & & & & & & \\
\hline 40 & FOOTPRINT-408-01 & H.Tec & Mami & 39071 & H.TeC & Twister, Excalbur,Brookdands & 1928 \\
\hline 41 & 1 POOTPRINT $415-01$ & Columbia & Blackrock, Thundersoorat & 19411 & Pyrenees & एา6 & 39143 \\
\hline 42 & 2: FOOTPRINT-422-01 & Joe Boxer & Skabe 47460 & $3 e 373$ & & & \\
\hline 43 & 3. FOOTPRINT $422-02$ & & & & & & \\
\hline 44 & 4 FOOTPRINT $423-01$ & Nhe & Air Max Deluxe E & 8150 & Nhe & Air Sentry Plus, Air Max Daro & 37560 \\
\hline 45 & SOOTPRINT $427-01$ & Wison & Prostaff 1000 , Prostaff 710 & 45614 & Wison & Drawbock & 45615 \\
\hline 46 & 5OOTPRINT $427-02$ & & & & & & \\
\hline 47 & 7) FOOTPRINT-427-03 & & & & & & \\
\hline 48 & POOTPRINT $428-01$ & Globe & Motto, Flux, Falcon & 21012 & & & \\
\hline 49 & 9: FOOTPRINT $430-01$ & KSwiss & Olten, Farias & 51185 & & & \\
\hline so & D FOOTPRINT - 434-01 & Frank Wright & Weller & 1753 & & & \\
\hline sit & 1 FOOTPAINT 435 OI & Thom MoN . & 84606 & 40962 & & & \\
\hline 2 & 2 FOOTPRTN 43502 & & & & & & \\
\hline 53 & 3: FOOTPRINT-438-0I & Lee & 2046 & 22801 & & & \\
\hline
\end{tabular}

Fig. 5. Ground Truth associated with crime scene images.

and labels. Assuming only up to pairwise clique potentials to be non-zero, the joint distribution over the labels $y=\left\{y_{1}, y_{2}, . ., y_{m}\right\}$ can be written as

$$
p(y \mid \mathbf{x})=\frac{1}{Z} \exp \left(\sum_{j} A_{j}\left(y_{j}, \mathbf{x}\right)+\sum_{(j, k) \in E} I_{j k}\left(y_{j}, y_{k}, \mathbf{x}\right)\right)
$$

where $Z$ is a normalizing constant known as the partition function and $A_{i}$ and $I_{i j}$ are the unary and pairwise potentials and $E$ are edges in the graph. Thus we can define the conditional probabilistic model

$$
P(y \mid \mathbf{x}, \theta)=\frac{e^{\psi(y, \mathbf{x} ; \theta)}}{\sum_{y^{\prime}} e^{\psi\left(y^{\prime}, \mathbf{x} ; \theta\right)}}
$$

where $\theta$ consists of the model parameters and $\psi(y, \mathbf{x} ; \theta) \in \mathcal{R}$ is a potential function defined as :

$$
\psi(y, \mathbf{x} ; \theta)=\sum_{j=1}^{m}\left(A\left(j, y_{j}, \mathbf{x} ; \theta^{s}\right)+\sum_{(j, k) \in E} I\left(j, k, y_{j}, y_{k}, \mathbf{x} ; \theta^{t}\right)\right)
$$

The first term in (3) is called the state term, sometimes called the association potential as mentioned in [37], and it associates the characteristics of that patch with its corresponding label. $\theta^{s}$ are called the state 


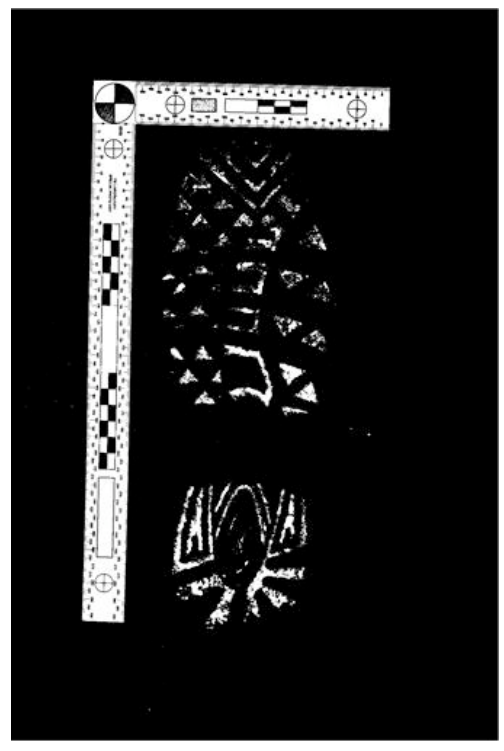

(a)

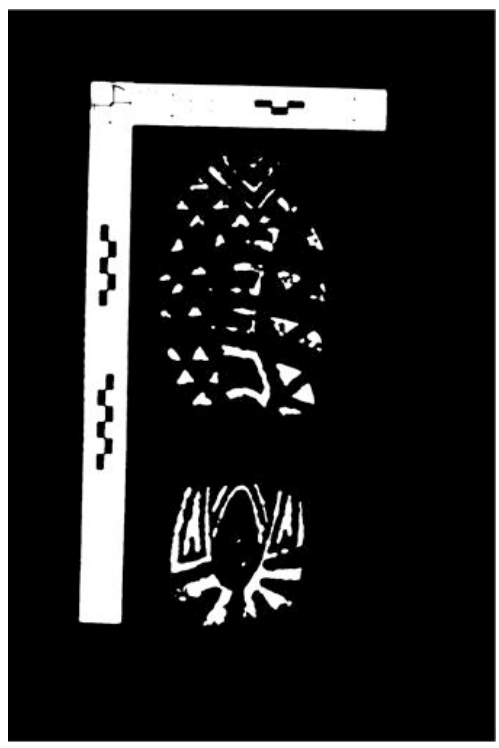

(b)

Fig. 6. Adaptive thresholding results: (a) crime scene image (b) enhanced image using adaptive thresholding.

parameters for the CRF model. Analogous to it, the second term in (3) called the interaction potential, captures the neighbor/contextual dependencies by associating pair wise interaction of the neighboring labels and the observed data. $\theta^{t}$ are called the transition parameters of the CRF model. $E$ is a set of edges that identify the neighbors of a patch; a 24-neighborhood model was used. $\theta$ comprises of the state parameters, $\theta^{s}$ and the transition parameters, $\theta^{t}$.

The association potential can be modeled as $A\left(j, y_{j}, \mathbf{x} ; \theta^{s}\right)=\sum_{i}\left(f_{i}^{s_{2}} \cdot \theta_{i j}^{s_{2}}\right)$ where $f_{i}^{s_{2}}$ is the $i^{\text {th }}$ state feature for that patch and $\theta_{i j}^{s_{2}}$ is a state parameter. The state features used will be described shortly. In order to introduce a non-linear decision boundary, the state features, $f_{i}^{s_{2}}$ are obtained by transforming the input features $f_{i}^{s_{1}}$ by the $\tanh$ function to give the transformed state feature $f_{i}^{s_{2}}=\tanh \left(\sum_{l}\left(f_{l}^{s_{1}}\left(j, y_{j}, \mathbf{x}\right) \cdot \theta_{i l}^{s_{1}}\right)\right)$ where $f_{l}^{s_{1}}$ is the $l^{\text {th }}$ state feature extracted for that patch; the transformed features are analogous to the outputs at the hidden layer of a neural network. The state parameters $\theta^{s}$ are a union of the two sets of parameters $\theta^{s_{1}}$ and $\theta^{s_{2}}$. The interaction potential $I(\cdot)$ is an inner product between the transition parameters $\theta^{t}$ and the transition features $f^{t}$ is as follows: $I\left(j, k, y_{j}, y_{k}, \mathbf{x} ; \theta^{t}\right)=\sum_{i}\left(f_{i}^{t}\left(j, k, y_{j}, y_{k}, \mathbf{x}\right) \cdot \theta_{i j k}^{t}\right)$.

Parameter Estimation. There are numerous ways to estimate the parameters of this CRF model [38]. In order to avoid the computation of the partition function the parameters are learnt by maximizing the likelihood of the data. Here we use conjugate gradient to maximize the likelihood. The maximum likelihood estimate of the parameters, $\theta$, based on a data set of size $M$ is given by

$$
\theta_{M L}=\underset{\theta}{\arg \max } \prod_{i=1}^{M} P\left(y_{i} \mid y_{N_{i}}, \mathbf{x}, \theta\right)
$$

where $P\left(y_{i} \mid y_{N_{i}}, \mathbf{x}, \theta\right)$, which is the probability of the label $y_{i}$ for a particular patch $i$ given the labels of its neighbors, $y_{N_{i}}$, is

$$
P(y \mid \mathbf{x}, \theta)=\frac{e^{\psi(y, \mathbf{x} ; \theta)}}{\sum_{a} e^{\psi\left(y_{i}=a, \mathbf{x} ; \theta\right)}}
$$

where $\psi\left(y_{i}, \mathbf{x} ; \theta\right)$ is defined by (3). Note that (4) has an additional $y_{N_{i}}$ in the conditioning set. This makes the factorization into products feasible as the set of neighbors for the patch from the minimal Markov blanket. It 
is also important to note that the resulting product only gives a pseudo-likelihood and not the true likelihood. The estimation of parameters which maximize the true likelihood may be very expensive and intractable for the problem at hand.

Combining (4) and (5), the log-likelihood is

$$
\left.\mathcal{L}(\theta)=\sum_{i=1}^{M}\left(\psi\left(y_{i}=a\right), \mathbf{x} ; \theta\right)-\log \sum_{a} e^{\psi\left(y_{i}=a, \mathbf{x} ; \theta\right)}\right) .
$$

The parameters are estimated by maximizing the log-likelihood function in (6) using gradient descent.

Features for $C R F$. Since features depend on whether the print is powder on a carpet, mud on a table etc, a general definition of the texture of footwear-prints is difficult. Thus an interactive design is used where the user provides the texture samples of the foreground and background from the image. The sample size is fixed to be $15 \times 15$ which is big enough to extract information and small enough to cover the print region. There could be one or more samples of foreground and background. The feature vector of these samples are normalized image histograms. There are four state features, the first two of which are derived from the probability distribution of gray levels in the patch: (i) entropy of the patch defined as $E(P)=-\sum_{i}^{n} p\left(x_{i}\right) * \log \left(p\left(x_{i}\right)\right.$ ), and (ii) standard deviation of the patch $S T D(P)=\sqrt{\sum_{i}^{n}\left(x_{i}-\mu\right)^{2}}$. The other two state features, which are based on cosine similarity between normalized image histogram vectors of two patches defined as $C S\left(P_{1}, P_{2}\right)=\frac{P_{1} * P_{2}}{\left|P_{1}\right|\left|P_{2}\right|}$, are: (iii) the cosine similarity between the patch and the foreground sample feature vectors and (iv) the cosine similarity between the patch and the background sample feature vectors. The transition feature is the cosine similarity between the current patch and the surrounding 24 patches.

Performance. Pixel labeling performance of several different algorithms are shown in Fig. 7. It includes an example input image and results from each of three methods: Otsu thresholding, a neural network and a CRF. Both the neural network and CRF models used the same feature set other than the transition feature. The input images were converted from RGB jpeg format to grayscale, with a resolution of $100 \mathrm{dpi}$, before processing. Overall performance on a data set of 45 images (11 hand-truthed prints yielding 320,000 $3 \times 3$ pathces for training and 34 images for testing), measured in terms of precision, recall and F-measure, are given in Fig. 7 (e). Precision, $\mathrm{P}$, is defined as the percentage of the extracted pixels which are correctly labeled as foreground(shoe-print). Recall, $\mathrm{R}$, is the percentage of the foreground successfully extracted. Fmeasure is the equally weighted harmonic mean of precision and recall i.e., $F=2 P R /(P+R)$. Performance of Otsu thresholding is poor if either the contrast between the foreground and the background is less or the background is inhomogeneous. The neural network performs a little better by exploiting the texture samples that the user provided. CRF tends to outperform both by exploiting the dependency between the current patch and its neighborhood, i. e., if a patch belongs to foreground but is ambiguous, the evidence given by its neighborhood patches helps in deciding its polarity.

Edge detection. Rather than labeling pixels in the gray-scale image to convert to a binary image, an alternative is to detect sharp discontinuities, or edges in the input image, as the starting point. Edge detection has a firm basis in biological vision and has been studied extensively. Edges in the image can be used to detect more global geometrical patterns as described in Section 4.4.

Among various edge detectors the Canny edge detector [39] has been shown to have many useful properties. It is considered to be the most powerful edge detector since it uses a multi-stage algorithm consisting of noise reduction, gradient calculation, non-maximal suppression and edge linking with hysteresis thresholding. The detected edges preserve the most important geometric features on shoe outsoles, such as straight line segments, circles, ellipses. The results of applying the Canny edge operator to crime scene images is shown in Fig. 8. Results with some database images are shown in Fig. 9.

Prior to edge detection, morphological operations are performed on database images [40]. The morphological operations are: dilation, erosion and filling holes in the binary image. The result is a more exact region boundary that improves the quality of edge detection. Morphological operations play a vital role in fetching the exact contours of the different shapes like line, ellipse and circle. We perform morphological operations 


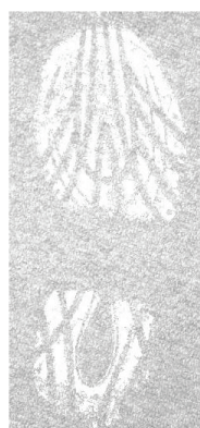

(a)

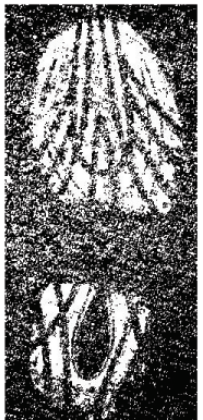

(b)

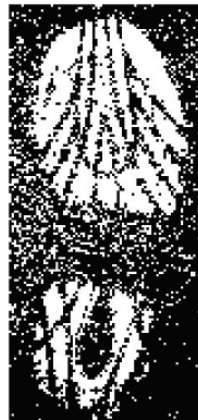

(c)

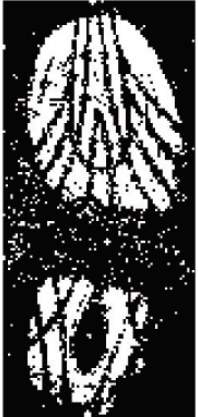

(d)

\begin{tabular}{|c|c|c|c|}
\hline Method & Precision & Recall & F-measure \\
\hline Otsu & 40.97 & 89.64 & 56.24 \\
\hline Neural Network & 58.01 & 80.97 & 59.53 \\
\hline CRF & 52.12 & 90.43 & 66.12 \\
\hline
\end{tabular}

(e) Summary Results

Fig. 7. Results of three image pixel labeling methods: (a) an input crime scene test image, (b) result obtained by applying Otsu thresholding, (c) result of neural network thresholding, and (d) result of CRF labeling. Summary of results with 34 test images are tabulated in (e) whose columns correspond to retrieval performance metrics (precision, recall and F-measure percentages).

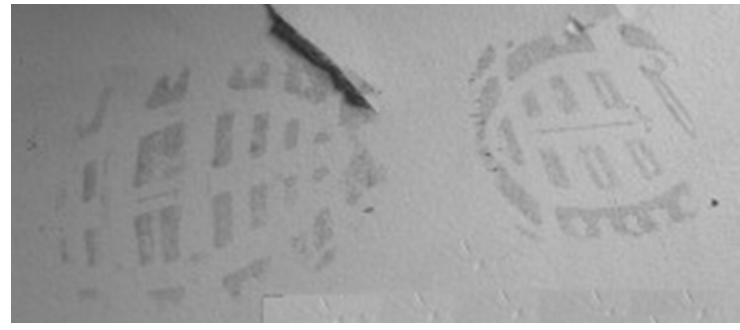

(a)

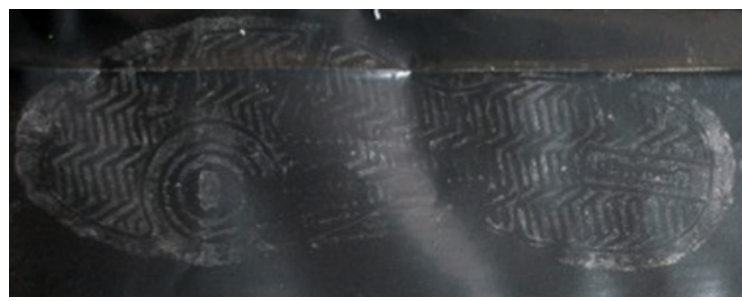

(c)

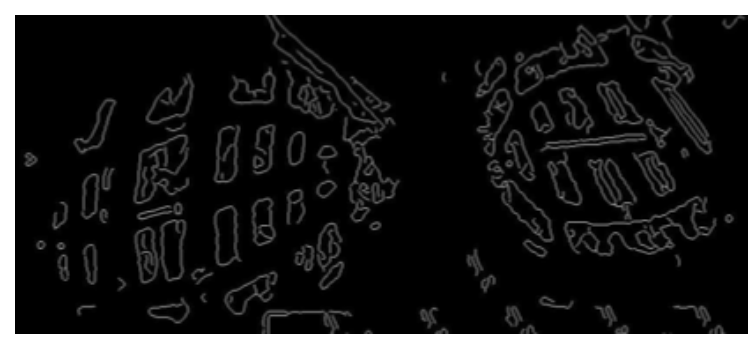

(b)

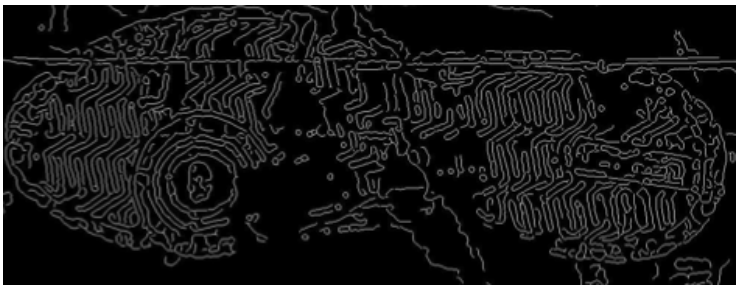

(d)

Fig. 8. Results of applying edge detection to crime scene images. Two pairs of images are shown corresponding to input and edge image: $(\mathrm{a}, \mathrm{b}),(\mathrm{c}, \mathrm{d})$. 

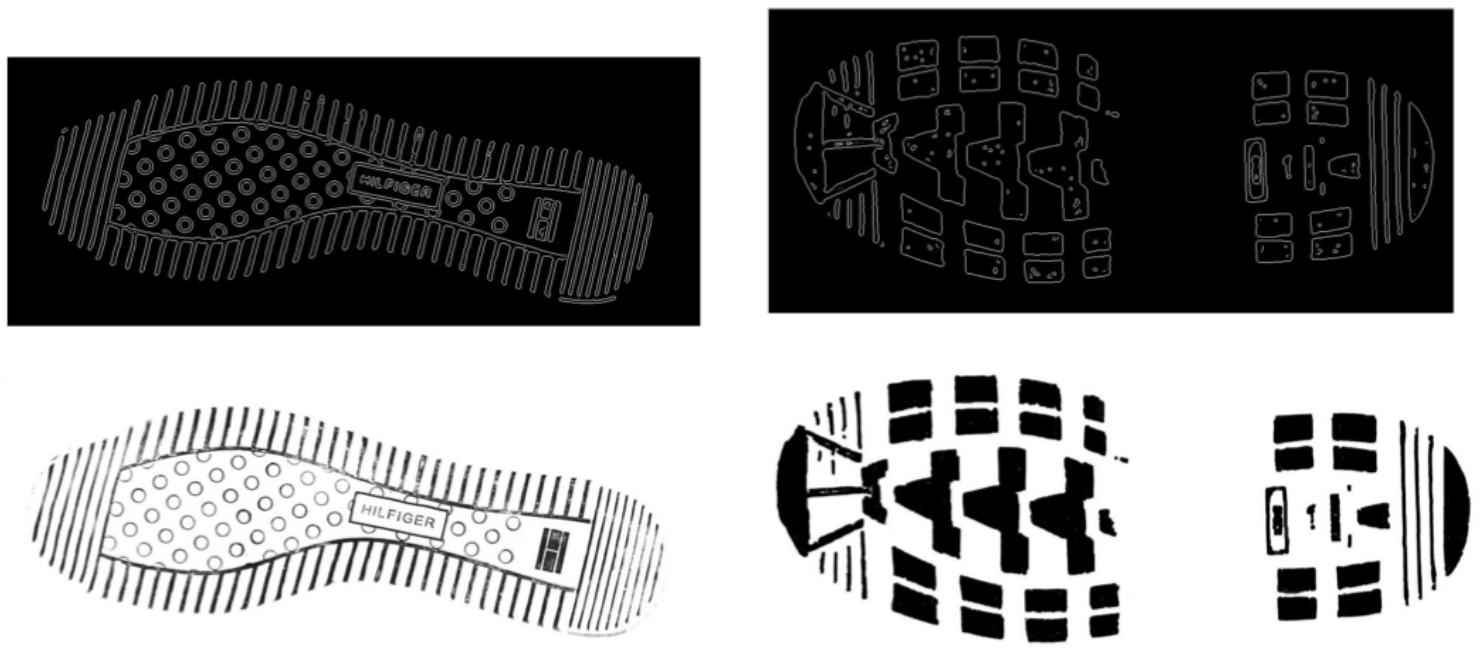

(a)

(b)

Fig. 9. Results of edge detection on two reference images.

(dilation and erosion) to make the interior region of the boundary uniform and then extract the boundary using Canny edge detection. Since the interior region is uniform, canny edge detector does not detect any edges inside the boundary and it improves the quality of edge detection. Specifically, each database shoe-print is processed in the following order: Edge detection $\rightarrow$ Dilation $\rightarrow$ Erosion $\rightarrow$ Flood fill $\rightarrow$ Complement. This procedure is illustrated using a sample print in the Fig. 10(a-f). As shown in Fig. 10(g), the edge image of the enhanced print has much better quality for feature extraction.

Dilation and erosion make the interior region of the boundary uniform and then extract the boundary using edge detection. Since the interior region is uniform the edge detector does not detect any edges inside the boundary. Edge detection showing the intermediate results of morphological operations is shown in Figure 11. Database Prints are subject to the sequence: Edge Detection, Morphological Operation and Edge Detection. Crime Scene Prints are subjected to only Edge Detection. For crime scene prints, because of their poor quality, we directly extract features from the edge image of original image. It takes $4-5$ seconds to process one image on a desktop computer.

\subsection{Characteristics of Outsole Patterns}

Discriminating characteristics of outsole patterns and footwear impressions can be classified into two categories: those acquired during the manufacturing process and those acquired from wear. Manufacturing features are those that come from the manufacturing process, which include design patterns and defects. Acquired features refer to attributes that develop during the lifetime of the footwear, such as wear pattern and damage.

As in any pattern comparison task, the first step for matching a query print against a reference print is a representation in terms of characteristics. The ideal representation would allow discrimination between different outsoles but also be invariant to various transformations such as rotation, translation, distortion and noise. Once a set of characteristics are determined there is also a need for a suitable measure of similarity between feature sets.

Color, texture and shapes of primitive elements are commonly used to recognize objects in computer vision [41]. However color is absent here since acquired impression prints are gray-scale images. Textures are sensitive to acquisition methods and susceptible to wear while shapes are resistant to wear and present over 


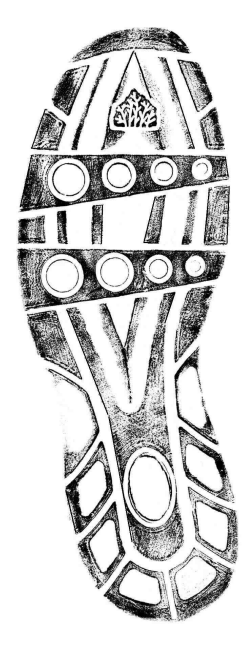

(a)

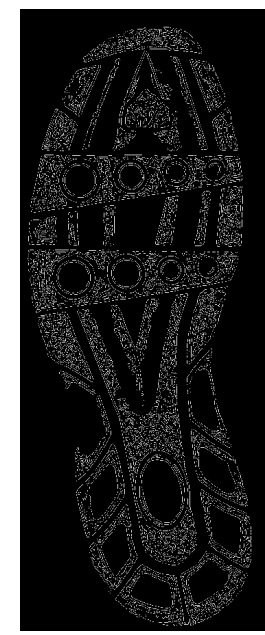

(b)

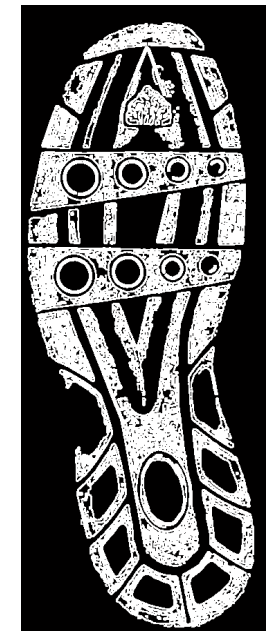

(c)

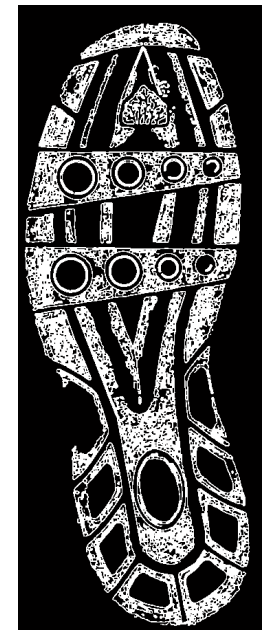

(d)

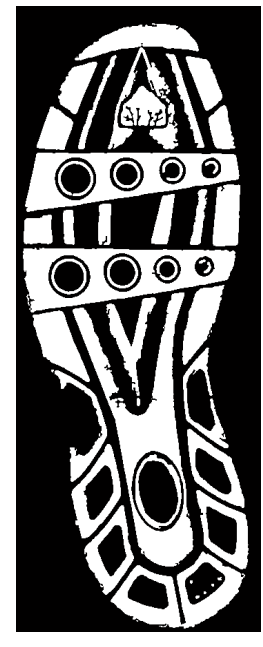

(e)

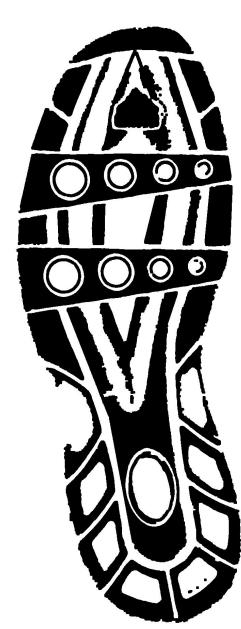

(f)

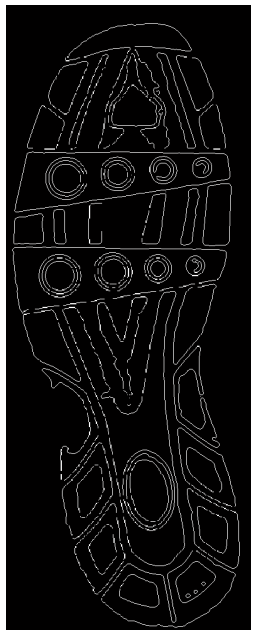

(g)

Fig. 10. Morphological operations for image enhancement: (a) input, (b) edge image, (c) after dilation, (d) after erosion, (e) after flood fill, (f) after complement, which is the final output, and (g) edge image of enhanced print. 

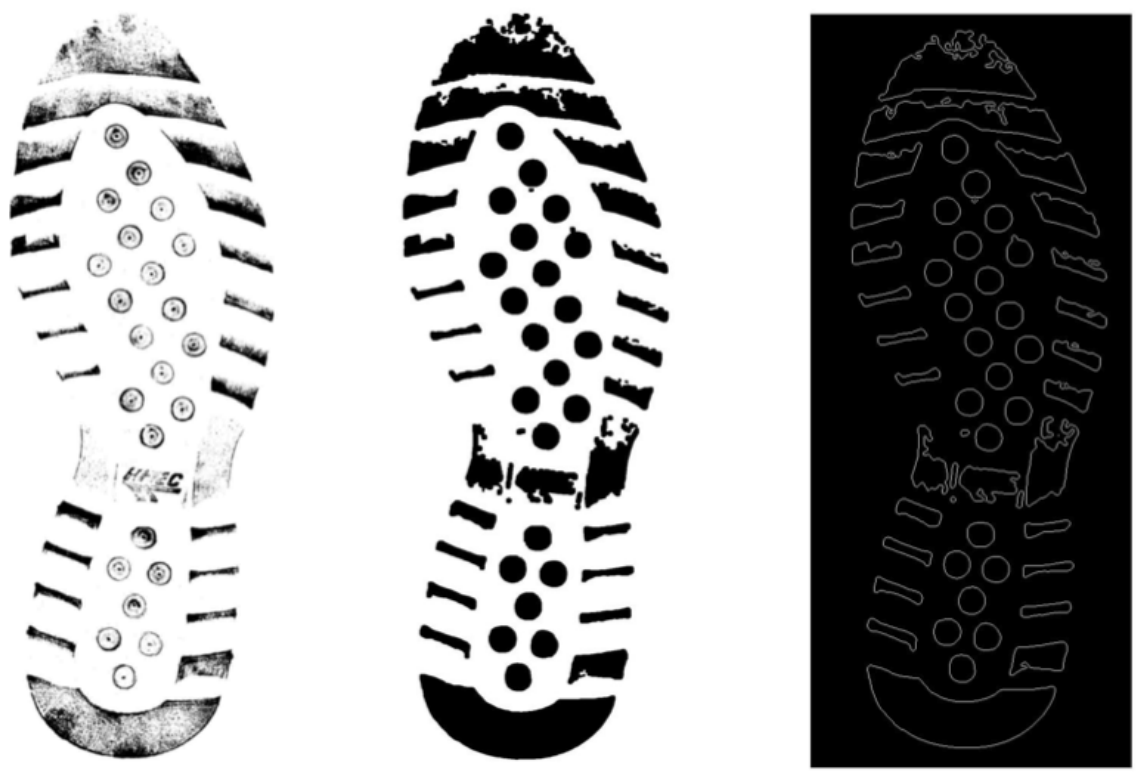

(a)
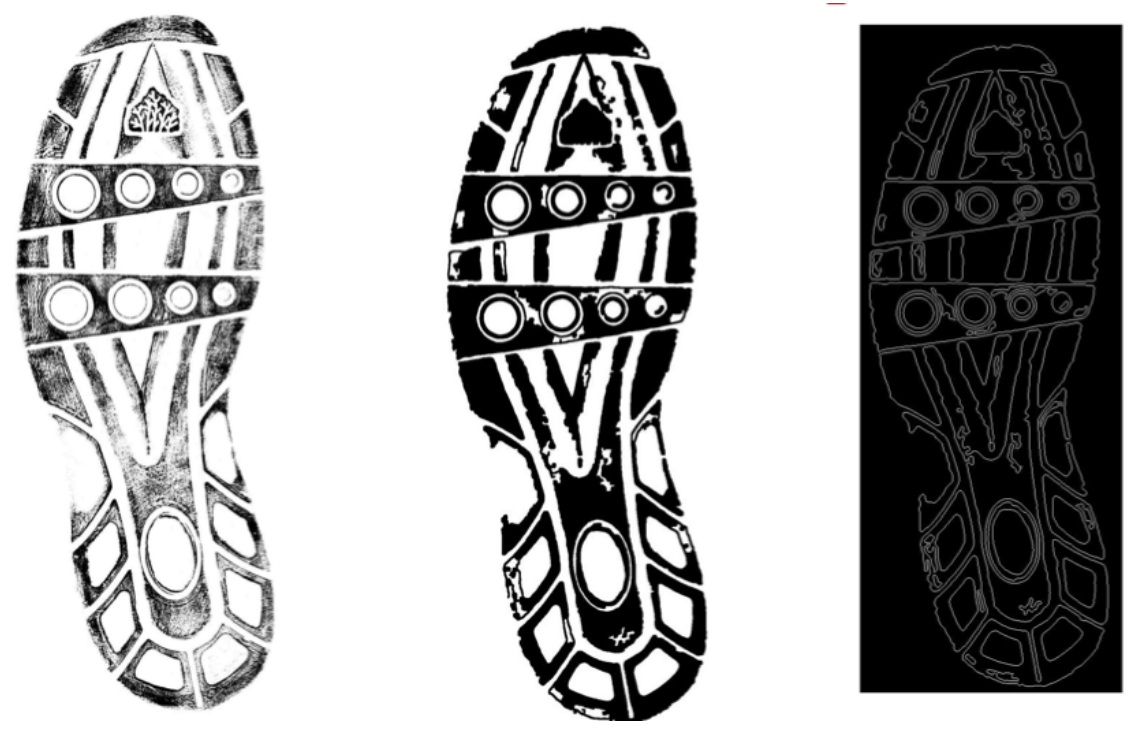

(b)

Fig. 11. Results of edge detection showing intermediate morphological operations on two data base images.

a long period of time. Shape features are also robust against occlusion and incompleteness, i.e., the wear or variation of a local region on the outsole will be less likely to affect shape features in other regions.

We discuss here three different types of characteristics for representing outsole patterns. Associated with each is a similarity measure for comparison of two inputs. The methods are GSC, SIFT and geometrical patterns represented as an attribute relational graph. 


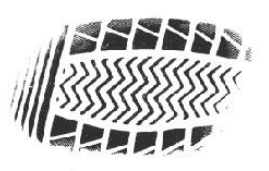

(a)

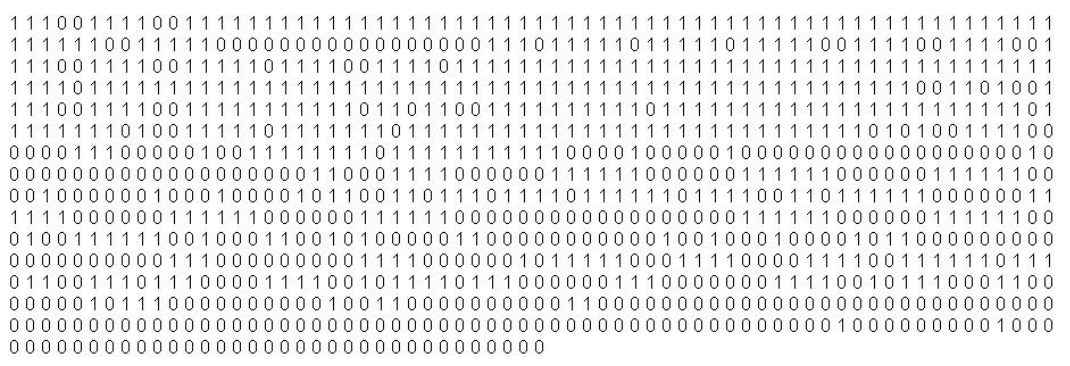

11111100111110000000000000000011101111101111101111100111100111100 11001111001111101111001111011111111111111111111111111111111111111

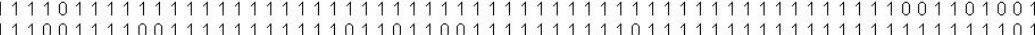
11111101001111101111111011111111111111111111111111111010100111100 000011100000100111111110111111111110000100000100000000000000000010 000000000000000000011000111100000011111100000011111100000011111100 001000000100010000101100110111011110111111011110011011111100000011 010011111100100011001010000011000000000001001000100001011000000000 00000000001110000000001111000000101111100011110000111100111111011 000001011100000000001001100000000001100000000000000000000000000000 0000000000000000000000000000000000

(b)

Fig. 12. Representation of an outsole pattern using features (GSC) designed for two-dimensional shapes in document analysis: (a) input impression which is characterized by (b) a 1,024-dimensional GSC binary feature vector.

GSC. In the field of document analysis, the central task is that of recognizing two-dimensional patterns such as characters. Many different features have been developed and one that we have used with success for handwriting recognition and writer verification are the GSC (gradient, structural, concavity) features. The GSC features are based on detecting local, intermediate and global features (see Fig. 12) [42]. The basic unit of an image is the pixel and we are interested in its relationships to neighbors at different ranges from local to global. In a sense, we are taking a multi-resolution approach to feature generation. GSC features are generated at three ranges: local, intermediate and global. In the basic approach the feature vector consists of 512 bits corresponding to gradient (192 bits), structural (192 bits), and concavity (128 bits) features. Each of these three sets of features rely on dividing the scanned image into a $4 \times 4$ region. Gradient features capture the frequency of the direction of the gradient, as obtained by convolving the image with a Sobel edge operator, in each of 12 directions and then thresholding the resultant values to yield a 192-bit vector. The structural features capture, in the gradient image, the presence of corners, diagonal lines, and vertical and horizontal lines, as determined by 12 rules. Concavity features capture, in the binary image, major topological and geometrical features including direction of bays, presence of holes, and large vertical and horizontal patterns. The input shoe- print is represented as two $4 \times 4$ regions or a fixed-dimensional (1028bit) binary feature vector. The similarity between two GSC feature vectors is computed using a correlation measure.

SIFT. In the field of computer vision a popular algorithm for detecting key features of three-dimensional objects in digital images is the scale invariant feature transform (SIFT) [43]. The objective of SIFT is to extract and describe invariant features from images that can be used to perform matching between different views of an object in a scene. Four major steps of the algorithm are: scale-space extrema detection, key point localization, orientation assignment and key-point descriptor construction. The scale space is constructed by convolving the input image with a Gaussian function and resampling the smoothed image. Maxima and minima are determined by comparing each pixel in the pyramid to its 26 neighbors(in a $3 \times 3$ cube). These maxima and minima in the scale space are called as key points, which are in turn described by a 128dimensional vector: a normalized description of gradient histogram of the region around that key-point. The number of key points detected by the SIFT algorithm varies from image to image. Key-points of a shoe-print image are shown in Fig. 13(a) where there are 15, 499 key-points. One such key-point descriptor is shown in Fig. 13(b). The similarity between two descriptors is computed using the Euclidean distance between two 128-dimensional vectors and the similarity between two images is the number of key-points that match. SIFT is commonly used in content-based image retrieval and is said to be used in Google image search.

Performance with GSC and SIFT. GSC features, which are designed for two-dimensional patterns, are very fast and work well with complete shoe-prints but break-down when prints are partial; a fix can be made by detecting whether the print is partial. SIFT features, which are designed for three-dimensional objects, 


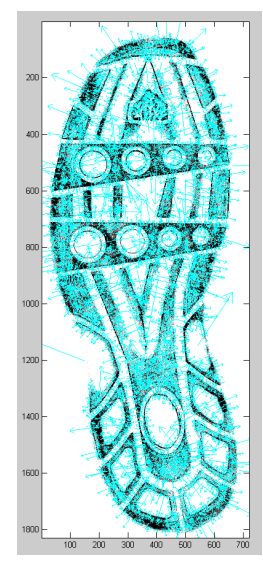

(a)

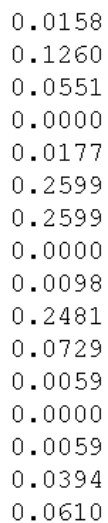

0.0610

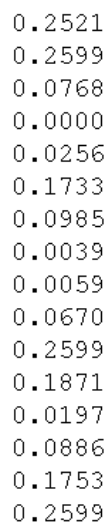

0.2599

$$
\begin{aligned}
& 0.0433 \\
& 0.0315 \\
& 0.0000 \\
& 0.0000 \\
& 0.0276 \\
& 0.0079 \\
& 0.0236 \\
& 0.0020 \\
& 0.0256 \\
& 0.1024 \\
& 0.2599 \\
& 0.0473 \\
& 0.0236 \\
& 0.1024 \\
& 0.1024 \\
& 0.0118
\end{aligned}
$$

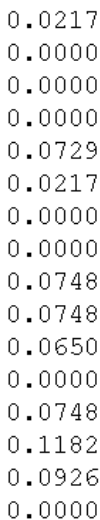

0.0217

0.0000

0.0729

0.021

0.0000

.0000

0.0748

0.0748

0.0748

0.0926

0.0000

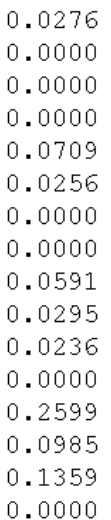

0.0276

0.0197

0.0020

0.0000

0.0000

0.0886

0.0236

0.0000

0.0000

0.1635

0.0118

0.0000

0.0000

0.0906

0.0039

0.0236

0.0000
0.0473

0.0039

0.0000

0.0000

0.1339

0.0138

0.0000

0.0000

0.1201

0.0374

0.0000

0.0000

0.0354

0.0059

0.0197

0.0039
0.0039

0.0000

0.0000

0.0000

0.0158

0.0729

0.0138

0.0000

0.0394

0.1674

0.0059

0.0000

0.0020

0.0098

0.0197

0.0079

(b)

Fig. 13. Representation of an outsole pattern using features (SIFT) designed for three-dimensional objects in computer vision: (a) input image annotated by key-points where each blue arrow shows key-point orientation extracted by the SIFT algorithm, and (b) descriptors for one key-point.

work better than GSC for partial prints, particularly since they were designed to handle occlusion in scenes. SIFT is invariant to transformations of scale, rotation and translation of shoe-prints [22]. However, due to local extrema in the scale space, SIFT key-points are not preserved both among different shoes of the same class and throughout the lifetime of a single shoe. A representation based on geometrical patterns in a graph works significantly better than SIFT in retrieval as described next (see Fig. 34).

\subsection{Geometrical patterns}

Patterns of outsoles usually contain small geometrical patterns involving short straight line segments, circles and ellipses. An analysis of 5,034 outsole prints revealed that $67 \%$ have only line segments (some examples are shown in Fig. 14, where the line segments have a minimum length of 25 pixels), $1.5 \%$ have only circles (Fig. 15), $0.004 \%$ have only ellipses (Fig. 16), and $24 \%$ are combinations of lines, circles and ellipses. The principal combination of shapes are lines-circles which constitute $16 \%$ (Fig. 17), lines-ellipses constitute $6 \%$ (Fig. 18), circles-ellipses-0.1\% (Fig. 19) and lines-circles-ellipses-0.7\% (Fig. 20). Texture patterns (Fig. 21) constitute the remaining $8 \%$. The complete distribution is given in Table 2 . This analysis shows that the three basic shapes are present in $92 \%$ of outsole prints. Furthermore, patterns other than circles and ellipses can be approximated by piecewise lines.

When projected on to a plane, most man-made objects can be represented as combinations of straight line and ellipse segments. Mathematically, straight line segments and circles are special cases of ellipses. An ellipse with zero eccentricity is a circle and an ellipse with eccentricity of 1 is a straight line; where the eccentricity of an ellipse is defined as $\sqrt{1-(b / a)^{2}}$ where $a$ and $b$ are the lengths of the semi-major and semi-minor axes.

While an ellipse detector alone can capture $92 \%$ of the primitive shapes, we choose to use specialized detectors for straight lines and circles since they are more efficient. The feature extraction approach is to detect the presence, location and size of three basic shapes: straight line segments, circles/arcs and ellipses. Since all three are geometrical shapes with simple parametric representations, they are ideal for the application of a robust method of detecting shapes.

The Hough transform[44] is a method to automatically detect basic geometrical patterns in noisy images. It detects features of a parametric form in an image by mapping foreground pixels into parameter space, which is characterized by an $n$ dimensional accumulator array, where $n$ is the number of parameters necessary 
Table 2. Distribution of geometric patterns in a database of footwear outsole prints.

\begin{tabular}{|c|c|}
\hline Fundamental Patterns & No. of Prints \\
\hline Line segments & 3397 \\
\hline Lines \& Circles & 812 \\
\hline Lines \& Ellipses & 285 \\
\hline Only Circles/Arcs & 73 \\
\hline Lines, Circles \& Ellipses & 37 \\
\hline Only Ellipses & 15 \\
\hline Circles \& Ellipses & 5 \\
\hline Texture & 410 \\
\hline \multicolumn{2}{|c|}{ Total - 5034 prints } \\
\hline
\end{tabular}

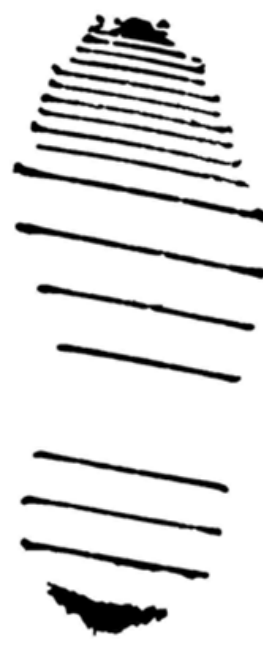

(a)

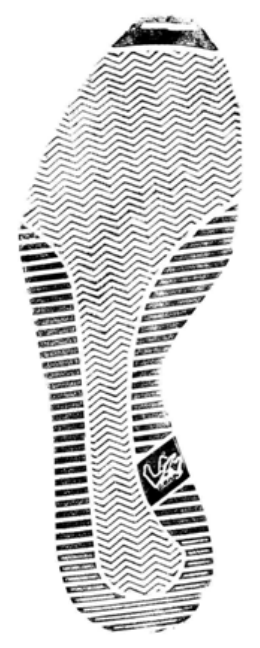

(b)

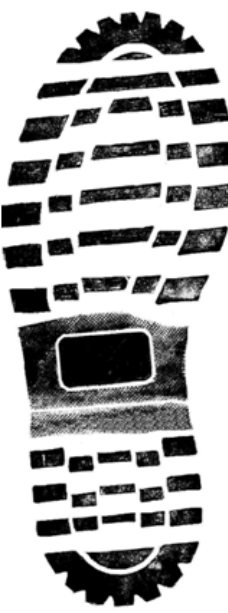

(c)

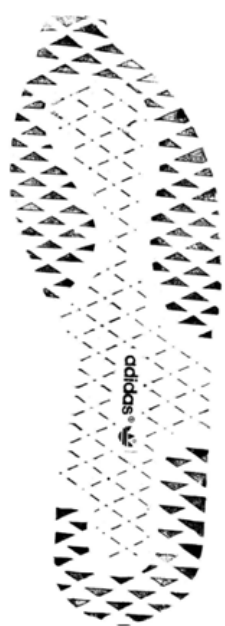

(d)

Fig. 14. Footwear outsole patterns containing line segments only. 


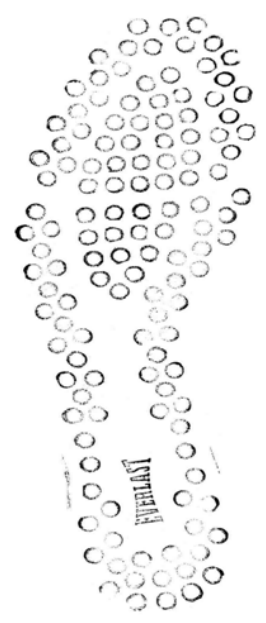

(a)

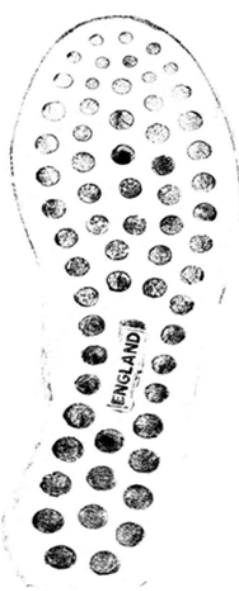

(b)

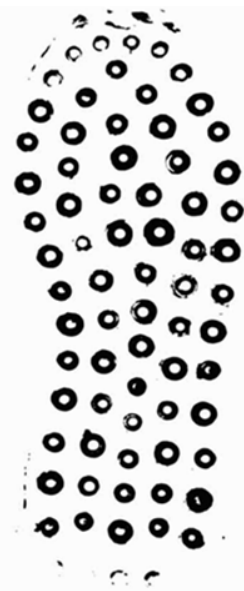

(c)

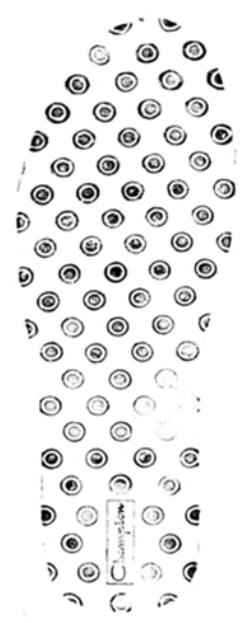

(d)

Fig. 15. Footwear outsole patterns containing circles only.

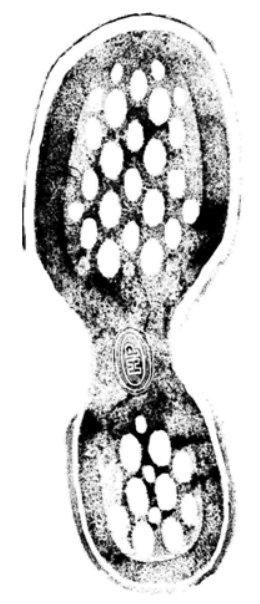

(a)

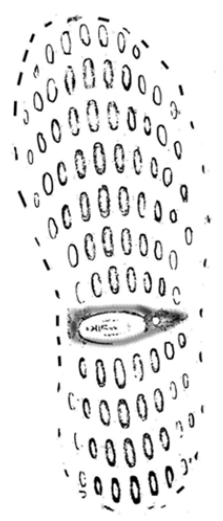

(b)

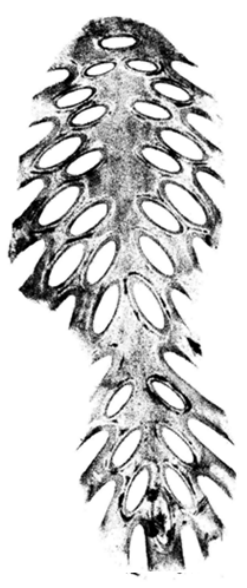

(c)

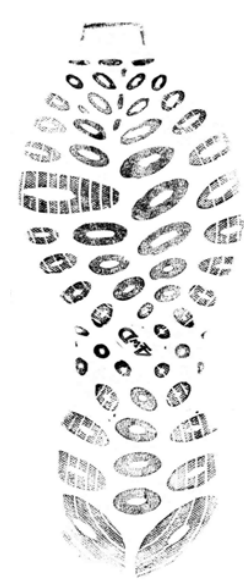

(d)

Fig. 16. Footwear outsole patterns containing ellipses only. 


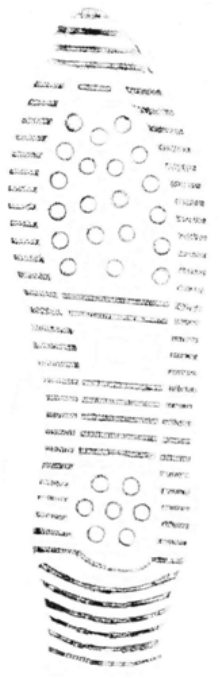

(a)

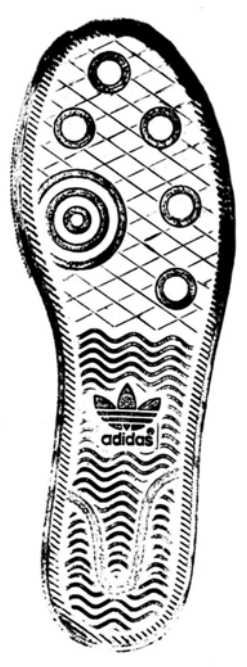

(b)

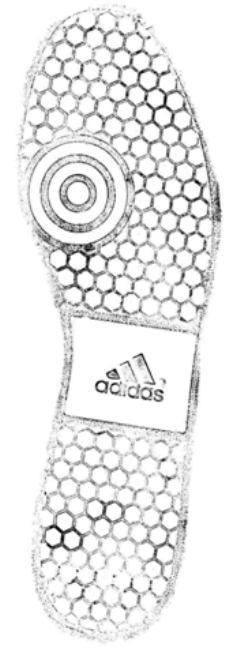

(c)

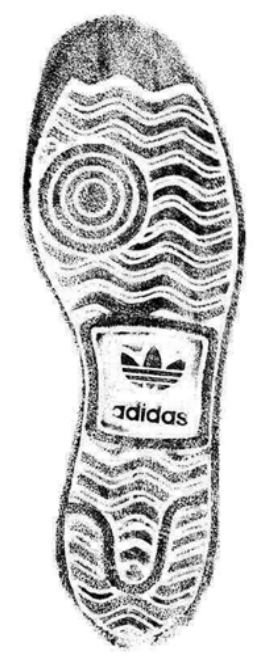

(d)

Fig. 17. Footwear outsole patterns containing lines and circles.

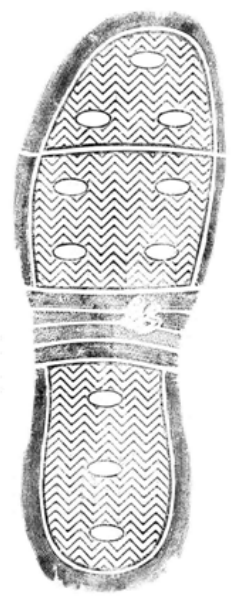

(a)

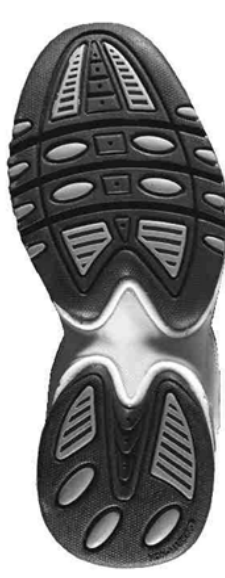

(b)

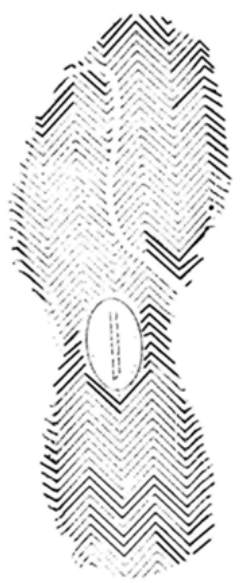

(c)

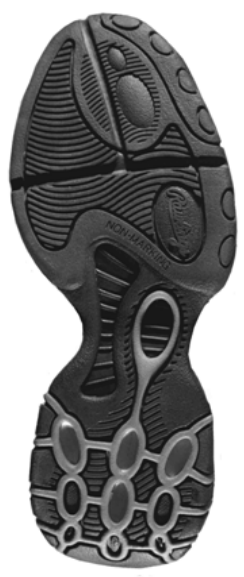

(d)

Fig. 18. Footwear outsole patterns containing lines and ellipses. 


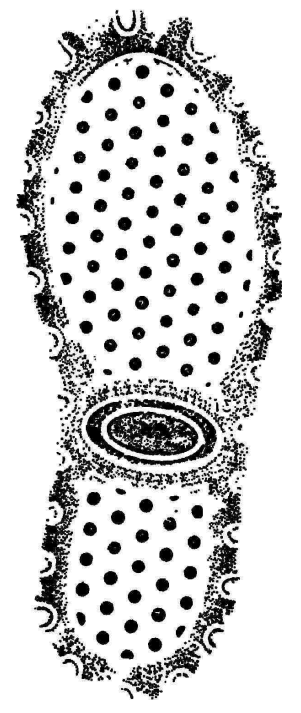

(a)

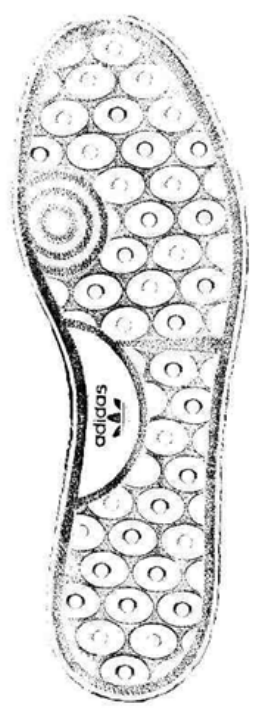

(b)

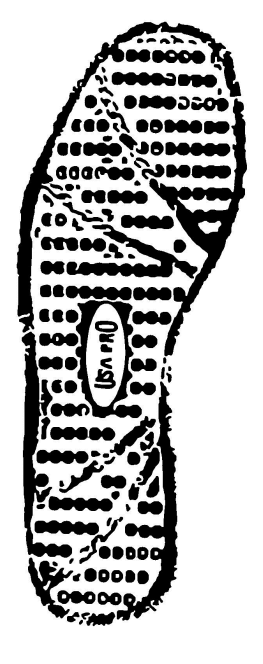

(c)

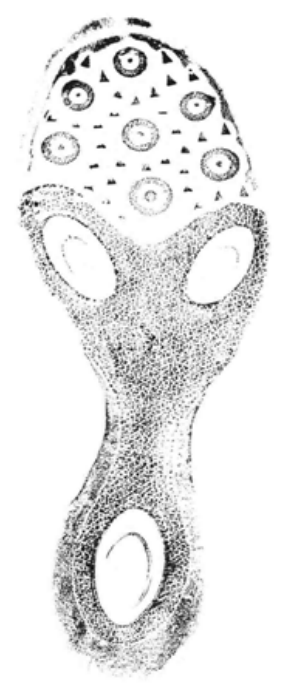

(d)

Fig. 19. Footwear outsole patterns containing circles and ellipses.

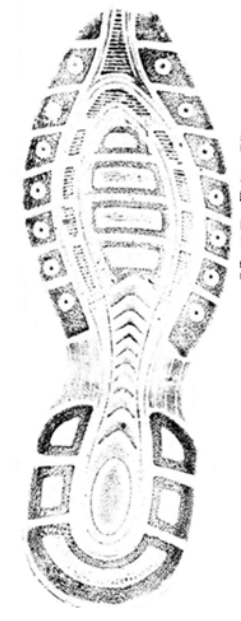

(a)

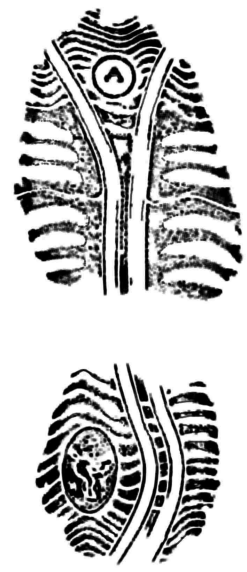

(b)

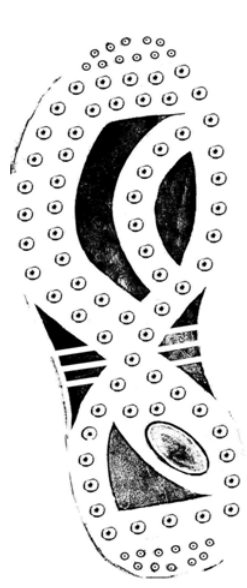

(c)

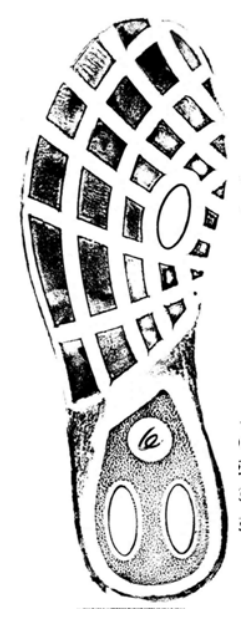

(d)

Fig. 20. Footwear outsole patterns containing lines, circles and ellipses. 


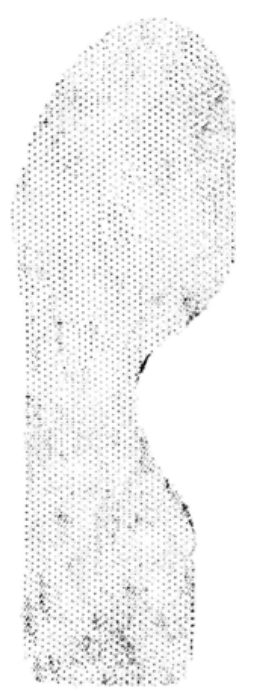

(a)

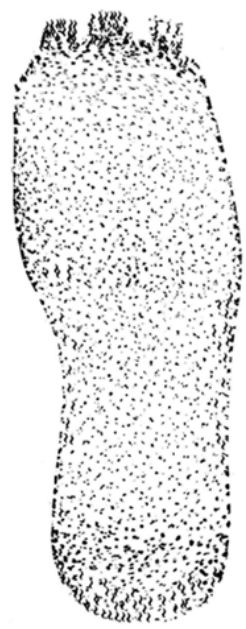

(b)

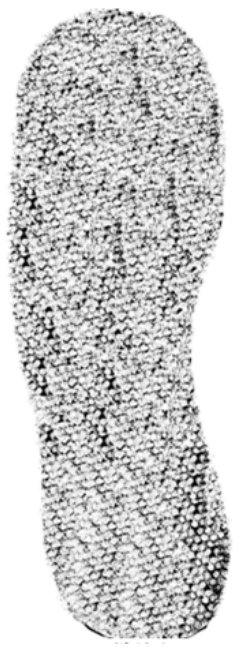

(c)
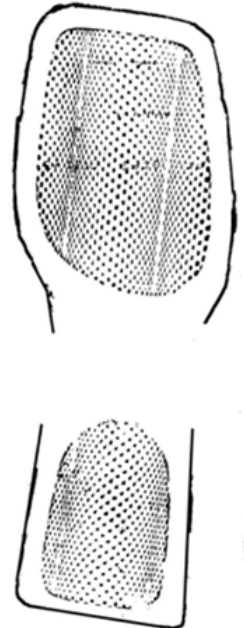

(d)

Fig. 21. Footwear outsole patterns containing texture only.

to describe the shape of interest. Each significant pixel from the shape of interest would cast a vote in the same cell of an accumulator array, hence all pixels of a shape gets accumulated as a peak. The number of peaks corresponds to the number of shapes of interest in the image. Originally designed for detecting straight lines in cloud chamber photographs and later generalized to circles and ellipses, the Hough transform has found success in many applications such as detecting cancerous nodules in radiological images and structure of textual lines in document images[45].

1. Line Segments: Using the polar coordinate system, a straight line can be represented by two parameters $r$ and $\theta$. The Hough transform maps each pixel in the Cartesian $x-y$ plane to a 2-dimensional accumulator array using the transformations defined by $x=r \cos \theta$ and $y=r \sin \theta$. The values of $r$ and $\theta$ at which the accumulator elements peak represent the presence of straight lines.

2. Circles: It involves building a 3-dimension accumulator array corresponding the center coordinates and the radius. Gradient orientation is used to limit the generation of spurious votes. Further, spatial constraints are used to identify spurious circles. Gradient orientation is used to limit the generation of spurious votes[46]. Further, spatial constraints are used to eliminate spurious circles.

3. Ellipses: In a Cartesian plane, an ellipse can be described by its centre $(p, q)$, length of the semi-major axis $a$, length of the semi-minor axis $b$ and the angle $\theta$ between the major axis and the $x$-axis. Thus five parameters $(p, q, a, b, \theta)$ are required to uniquely describe an ellipse[47]. These five parameters demand a five-dimensional accumulator which is computationally expensive but the Randomized Hough transform (RHT) [48] for ellipse detection is more efficient.

We describe next algorithms for lines and ellipses based on the Hough transform; since the circle is a special case of the ellipse the same algorithm can be used.

Line Detection. The Standard Hough Transform (SHT) to detect lines consists of three steps: transform and accumulation, peak selection, and line verification. However, most scenes have complex geometric structures. The number of line segments in a scene image of moderate size (say $1000 \times 1000$ ) can be several hundred. Each set of collinear points votes for a peak in accumulator. Detecting all the true peaks accurately while suppressing spurious ones is difficult. In addition, short line segments are easily missed, which may 
be useful for discriminating similar print structures. Since the standard Hough transform (SHT) cannot be applied directly, an iterative procedure is used to remove interference in peak selection, using a verification criterion. First, connected components are labeled in the edge image. For each component, the Hough transform is applied and peaks are detected. When a peak is identified and the line segments are extracted, pixels contributing to those line segments are eliminated from the edge image, and an updated accumulator is obtained by applying SHT on the modified edge image. The process of extracting straight line segments in a crime scene impression is shown in Figures 22.

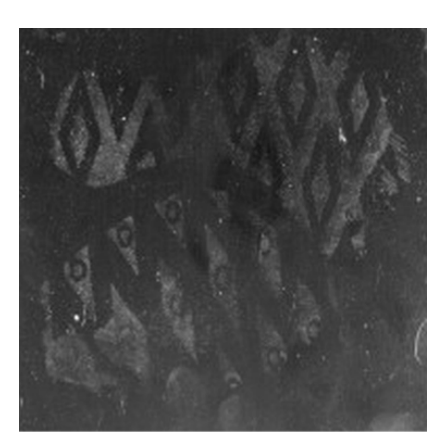

(a)

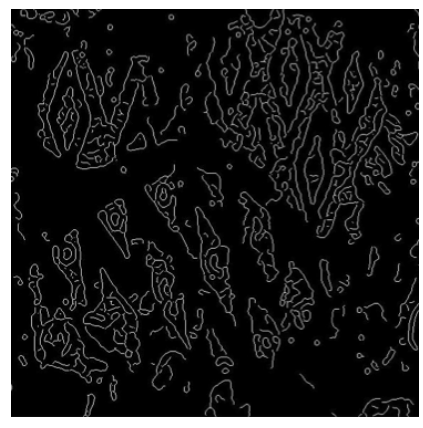

(b)

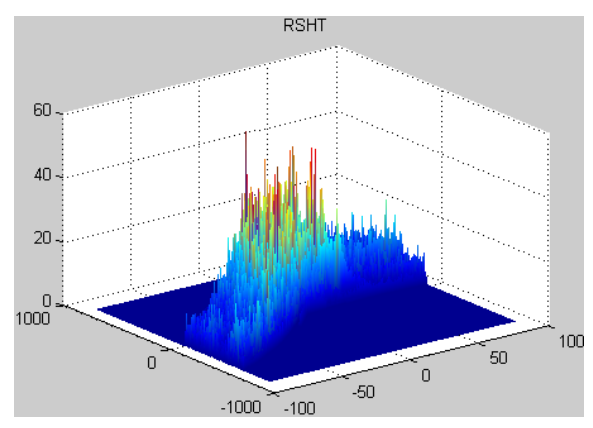

(c)

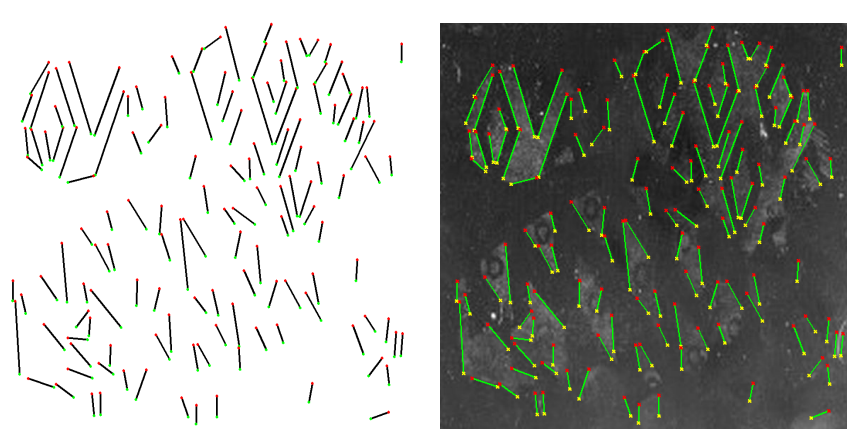

(d)

(e)

Fig. 22. Detecting line segments using the restricted straight line Hough transform: (a) input crime scene image, (b) edge detected image (c) accumulator histogram, (d) detected line segments, and (e) line segments overlaid on the input image.

Ellipse detection. The ellipse is a fundamental shape in both natural and man-made objects and hence frequently encountered in images. Existing ellipse detection algorithms, viz., randomized Hough transform (RHT) and multi-population genetic algorithm (MPGA), have disadvantages. The RHT performs poorly with multiple ellipses and MPGA has a high false positive for complex images. The proposed algorithm selects random points using constraints of smoothness, distance and curvature. In the process of sampling, parameters of potential ellipses are progressively learnt to improve parameter accuracy. New probabilistic fitness measures are used to verify ellipses extracted: ellipse quality based on the Ramanujan approximation and completeness. Experiments on synthetic and real images show performance better than RHT and MPGA in detecting multiple, deformed, full or partial ellipses in the presence of noise and interference. A detailed description of the algorithm is given in [19].

Results of extracting circles and ellipses in data base prints are shown in 23. 


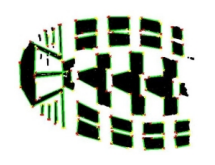

(a)

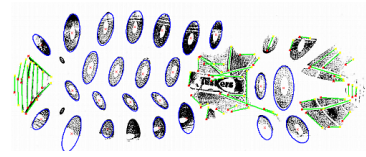

(d)

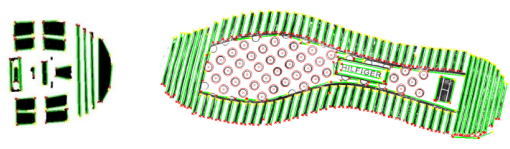

(b)

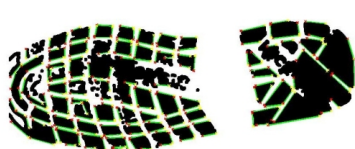

(c)

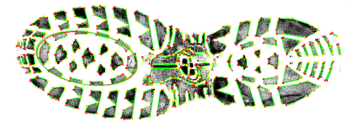

(e)

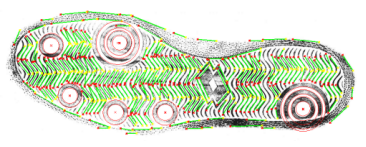

(f)

Fig. 23. Shapes detected in reference images: lines, circles and ellipses are shown in green, red and blue respectively.

\subsection{Graph representation}

Structural representations have long been used in computer vision to represent complex objects and scenes for image matching [49]. Graph representations have a great advantage over feature vectors because of they can explicitly model the relationship between different parts and feature points [50].

After detecting their presence, the impression image is decomposed into a set of primitives. To obtain a structural representation of these primitives, an attributed relational graph $(A R G)[51,52]$ is built. An $A R G$ is a directed graph that can be represented as a 3 -tuple $(V, E, A)$ where $V$ is the set of vertices, also called nodes, $E$ is the set of relations (edges) and $A$ is the set of attributes. Each edge describes the spatial relationship between a pair of nodes. The attributes include node attributes (unary) and edge attributes (binary).

There are three types of nodes, corresponding to lines (L), circles (C) and ellipses (E), and nine types of edges: line-to-line (L2L), line-to-circle (L2C), line-to-ellipse (L2E), circle-to-circle (C2C), circle-to-ellipse $(\mathrm{C} 2 \mathrm{E})$, ellipse-to-ellipse (E2E), circle-to-line $(\mathrm{C} 2 \mathrm{~L})$, ellipse-to-line (E2L) and ellipse-to-circle (E2C). Attributes of nodes and edges should be defined such that they are scale/rotation invariant, and capture spatial relationships such as distance, relative position, relative dimension and orientation.

Three attributes are defined for nodes which represent the basic shapes detected.

1. Quality is the ratio of the number of points on the boundary of the shape (perimeter pixels) to the perimeter of the shape.

2. Completeness is the standard deviation of the angles of all on-perimeter pixels with respect to the center of circle/ellipse, stdd, normalized as stdd/180. If a wide range of angles are present, implying that most of the shape is represented, there will be more angles represented and this value is high, while a partial figure will have smaller diversity of angles and this value will be low. While the range of angles is 0 to 360 for circles and ellipses, for a straight line there are only two angles with respect to the center, 0 and 180.

3. Eccentricity is the degree of elongation, defined as the square root of 1 minus square of ratio of minor to major axes. For a circle eccentricity is 0 and for a straight line eccentricity is 1 .

Edge attributes are dependent upon the pair of shapes they connect. They use the relative position definitions between lines, circles and ellipses. Some attributes are normalized to the range $[0,1]$ using the sigmoid function. A complete list of node and edge attributes is given in Figure 24.

So as to handle missing nodes or incorrectly detected nodes, which may arise due to noise, occlusion and incompleteness, a fully-connected graph is used. If for the sake of computational efficiency we consider only local relationships, as is often done in Markov models, it would lead to poor results since the only image components discernible in a print may be those at the extremities.

This means that there is a directed edge from each node to all nodes including itself; a node is connected to itself because we can use a general formula for computing the cost between two graphs. Thus in a directed 
graph with $N$ nodes there will be $N+2(N(N-1) / 2)=N^{2}$ edges. The number of attributes at each edge depends on the types of nodes it connects.

The ARG for a scene image is shown in Fig. 25; the values of node and edge attributes for a portion of the subgraph with four nodes are given in Table 3.

Table 3. Node and Edge Attributes for four-node subgraph shown in Figure 25(d).

\begin{tabular}{|c|c|}
\hline Nodes and Edges & Attributes \\
\hline Node 1 & {$[0.0000,0.7468,0.5699]$} \\
\hline Node 2 & N $/ \mathrm{A}$ \\
\hline Node 3 & $\mathrm{~N} / \mathrm{A}$ \\
\hline Node 4 & $\mathrm{~N} / \mathrm{A}$ \\
\hline$E_{11}$ & {$[0.5000,0.0000,0.0000]$} \\
\hline$E_{12}$ & {$[0.4285,0.1976,0.5989]$} \\
\hline$E_{13}$ & {$[0.4809,0.1376,0.3195]$} \\
\hline$E_{14}$ & {$[0.5715,0.1976,0.5989]$} \\
\hline$E_{21}$ & {$[0.0000,0.5000,0.0000,0.0000,0.0200]$} \\
\hline$E_{22}$ & {$[0.0000,0.5312,0.0584,0.0000,0.0200]$} \\
\hline$E_{23}$ & {$[0.0323,0.5527,0.0609,0.0146,0.0626]$} \\
\hline$E_{24}$ & {$[0.5407,0.1976,0.3195]$} \\
\hline$E_{31}$ & {$[0.0000,0.4688,0.0584,0.0000,0.0200]$} \\
\hline$E_{32}$ & {$[0.0000,0.5000,0.0000,0.0000,0.0200]$} \\
\hline$E_{33}$ & {$[0.0324,0.5217,0.0091,0.0090,0.1018]$} \\
\hline$E_{34}$ & {$[0.5191,0.1387,0.2316]$} \\
\hline$E_{41}$ & {$[0.0323,0.4473,0.0609,0.0085,0.0901]$} \\
\hline$E_{42}$ & {$[0.0324,0.4783,0.0091,0.0091,0.0903]$} \\
\hline$E_{43}$ & {$[0.0000,0.5000,0.0000,0.0000,0.0200]$} \\
\hline$E_{44}$ & \\
\hline &
\end{tabular}

\subsection{Graph Similarity}

Central to both retrieval and identification is a method for computing similarity between images. Equivalently, the inverse of similarity is a distance measure. The choice of similarity or distance measure is important since it influences the retrieval result, uncertainty of match, and the quality of clusters in partitioning the database for efficiency.

Image retrieval applications typically employ histogram (or probability density) distance measures. Binby-bin distance measures such as Euclidean distance (or its generalization known as the Minkowski distance) and Kullback-Leibler divergence are perceptually unsatisfactory. Earth Mover's Distance (EMD), a cross bin distance metric is popular in content-based image retrieval [53]. Advantages of EMD are that it allows partial matches, ability to efficiently handle high-dimensional feature spaces and closeness to perceptual similarity when applied to image histograms.

Earth Mover's Distance. EMD evaluates the least amount of work that is needed to transform one distribution into the other. Consider the evaluation of the distance between two signatures (histograms) $P_{1}=\left\{P_{1 i} \mid 1 \leq i \leq n_{1}\right\}$ and $P_{2}=\left\{P_{2 j} \mid 1 \leq j \leq n_{2}\right\}$. The bins $\left[P_{1 i}\right]$ have corresponding weights $\mathbf{w}_{1}=\left[w_{1 i}\right]$ and similarly $\left[P_{2 j}\right]$ have weights $\mathbf{w}_{2}=\left[w_{2 j}\right]$. The ground distance matrix $\mathbf{C}=\left[c_{i j}\right]$ specifies ground distance between all pairs of bins, $c_{i j}$. The flow matrix $\mathbf{F}=\left[f_{i j}\right]$, where $f_{i j}$ is the amount of "supplies" transferred from bin $P_{1 i}$ to bin $P_{2 j}$. The goal is to find proper values of $\mathbf{F}$ in order to minimize the overall work given by

$$
\operatorname{WORK}\left(\mathbf{w}_{1}, \mathbf{w}_{2}, \mathbf{C}\right)=\sum_{i=1}^{n_{1}} \sum_{j=1}^{n_{2}} c_{i j} f_{i j}
$$

which is subject to the following constraints:

$$
\begin{gathered}
f_{i j} \geq 0, \quad \forall 1 \leq i \leq n_{1}, 1 \leq j \leq n_{2}, \\
\sum_{j=1}^{n_{2}} f_{i j} \leq w_{1 i}, \forall 1 \leq i \leq n_{1},
\end{gathered}
$$


L2L

\begin{tabular}{|c|c|c|c|}
\hline Att & Definition & Normalization & Weight \\
\hline \multirow{2}{*}{$\mathrm{N}-\boldsymbol{\alpha}$} & $|L 1 . \theta-L 2 . \theta|$ & \multirow[b]{2}{*}{ - } & \multirow{2}{*}{0.4472} \\
\hline & 180 & & \\
\hline \multirow{2}{*}{$N-r s$} & L1.len & & \multirow{2}{*}{0.4472} \\
\hline & $\overline{\text { L1.len +L2.len }}$ & - & \\
\hline \multirow{2}{*}{$r d$} & $\operatorname{dist}(L 1 . m, L 2 . m)$ & $r d$ & \multirow{2}{*}{0.4472} \\
\hline & L1.len +L2.len & $\sqrt{1+r d^{2}}$ & \\
\hline \multirow{2}{*}{$p d$} & $\operatorname{dist}(L 1 . m, L 2)$ & $p d$ & \multirow{2}{*}{0.4472} \\
\hline & L1.len + L2.len & $\sqrt{1+p d^{2}}$ & \\
\hline \multirow{2}{*}{$r p_{1}$} & $\min (O A, O B)$ & $r p_{1}+1$ & \multirow{2}{*}{0.4472} \\
\hline & $\max (O A, O B)$ & 2 & \\
\hline \multirow{2}{*}{$r p_{2}$} & $\min (|O A|,|O B|)$ & $r p_{2}+1$ & \multirow{2}{*}{0.4472} \\
\hline & $\overline{\max (|O A|,|O B|)}$ & 2 & \\
\hline
\end{tabular}

$\mathrm{C} 2 \mathrm{E}$

\begin{tabular}{|c|c|c|c|}
\hline N-rs & $\frac{C . r}{C . r+E . E R}$ & - & 0.5774 \\
\hline$r d$ & $\frac{\operatorname{dist}(\text { C.cen, E.cen })}{\text { C.r }+ \text { E.ER }}$ & $\frac{r d}{\sqrt{1+r d^{2}}}$ & 0.5774 \\
\hline$r p$ & $\frac{\min (O A, O B)}{\max (O A, O B)}$ & $\frac{r p+1}{2}$ & 0.5774 \\
\hline
\end{tabular}

\section{E2 E}

\begin{tabular}{|c|c|c|c|}
\hline e_ratio & $\frac{\mathrm{E} 1 . \mathrm{e}}{\text { E1.e }+ \text { E2.e }}$ & - & 0.2236 \\
\hline$f(\Delta e)$ & $\frac{\text { E1. } \mathrm{e}-\mathrm{E} 2 . \mathrm{e}+\mathbf{1}}{2}$ & - & 0.2236 \\
\hline$r d$ & $\frac{\operatorname{dist}(\text { E1.cen, E2.cen })}{\text { E1.ER - E2. ER }}$ & $\frac{r d}{\sqrt{1+r d^{2}}}$ & 0.4472 \\
\hline$N-r s$ & $\frac{\text { E1. ER }}{\text { E1. ER + E2. ER }}$ & (2) & 0.4472 \\
\hline N-ro & $\begin{array}{c}|E 1 . \theta-E 2 . \theta| \\
90\end{array}$ & - & 0.4472 \\
\hline$r p$ & $\begin{array}{c}\text { rp(E1.major-axis, } \\
\text { E2.major-axis) }\end{array}$ & $\frac{r p+1}{2}$ & 0.4472 \\
\hline
\end{tabular}

L2E

\begin{tabular}{|c|c|c|c|}
\hline Att & Definition & Normalization & Weight \\
\hline N-rs & $\frac{\text { L.len }}{L . \text { len }+E . E R}$ & - & 0.5 \\
\hline$r d$ & $\frac{d i s t(L . m, E . c e n)}{L . \text { len }+E . E R}$ & $\frac{r d}{\sqrt{1+r d^{2}}}$ & 0.5 \\
\hline$r p 1$ & $\frac{\min (O A, O B)}{\max (O A, O B)}$ & $\frac{r p_{1}+1}{2}$ & 0.5 \\
\hline$r p 2$ & $-\frac{\min (|O A|,|O B|)}{\max (|O A|,|O B|)}$ & $\frac{r p_{2}+1}{2}$ & 0.5 \\
\hline$N-r o$ & $\frac{|L . \theta-E . \theta|}{180}$ & - & 0.5 \\
\hline
\end{tabular}

C2L

\begin{tabular}{|c|c|c|c|}
\hline N-rs & $\frac{\text { C.r }}{\text { C.r+I.len }}$ & - & 0.5774 \\
\hline$r d$ & $\frac{d i s t(C . c e n, L)}{C . r}$ & $\frac{r d}{\sqrt{1+r d^{2}}}$ & 0.5774 \\
\hline$r p$ & $\frac{\min (S 1, S 2)}{\max (S 1, S 2)}$ & $\frac{r p+1}{2}$ & 0.5774 \\
\hline
\end{tabular}

L2C

\begin{tabular}{|c|c|c|c|}
\hline N-rs & $\frac{\text { L.len }}{\text { C.r }+ \text { L.len }}$ & - & 0.5774 \\
\hline$r d$ & $\frac{d i s t(C . c e n, L)}{C . r}$ & $\frac{r d}{\sqrt{1+r d^{2}}}$ & 0.5774 \\
\hline$r p$ & $\frac{\min (S 1, S 2)}{\max (S 1, S 2)}$ & $\frac{r p+1}{2}$ & 0.5774 \\
\hline
\end{tabular}

\begin{tabular}{|c|c|c|c|}
\hline \multicolumn{3}{|c}{$\mathrm{C2C}$} \\
\hline$r-r s$ & $\frac{C 1 . r}{C 1 . r+C 2 . r}$ & - & 0.7071 \\
\hline$r d_{1}$ & $\frac{\operatorname{dist}(C 1 . c e n, C 2 . c e n)}{C 1 . r+C 2 . r}$ & $\frac{r d_{1}}{\sqrt{1+r d_{1}{ }^{2}}}$ & 0.7071 \\
\hline$r d_{2}$ & $\frac{\operatorname{dist}(C 1 . c e n, C 2 . c e n)}{C 1 . r-C 2 . r}$ & $\frac{r d_{2}}{\sqrt{1+r d_{2}{ }^{2}}}$ & 0.0 \\
\hline
\end{tabular}

\section{Node Attributes}

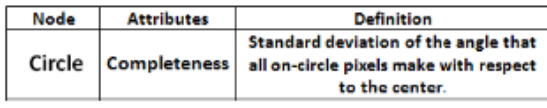

\begin{tabular}{|c|c|c|}
\hline Node & Attributes & Definition \\
\hline Circle & Quality & $\frac{\text { Number of pixels on circle }}{\text { Circumference of circle }}$ \\
\hline
\end{tabular}

\begin{tabular}{|c|c|c|}
\hline Node & Attributes & Definition \\
\hline Ellipse & Eccentricity & $\sqrt{1-\frac{b^{2}}{a^{2}}}$ \\
\hline
\end{tabular}

\section{Symbols and its Definition}

\begin{tabular}{|c|c|c|c|c|c|c|c|c|c|c|c|c|c|}
\hline $\mathbf{L}$ & $\begin{array}{c}\text { Line } \\
\text { segment }\end{array}$ & $r$ & Radius & e & eccentricity & C & Circle & len & Length & att & attributes & $\max$ & maximum \\
\hline E & Ellipse & $\mathrm{m}$ & Mid-point & cen & Centre & pd & $\begin{array}{c}\text { Perpendicular } \\
\text { distance }\end{array}$ & ER & $\sqrt{a \cdot b}$ & $\mathrm{~N}$ & Normalized & II & absolute \\
\hline dist & $\begin{array}{c}\text { Euclidean } \\
\text { distance }\end{array}$ & rp & $\begin{array}{l}\text { Relative } \\
\text { position }\end{array}$ & rd & $\begin{array}{l}\text { Relative } \\
\text { distance }\end{array}$ & rs & Relative size & $\theta$ & orientation & $a, b$ & $\begin{array}{l}\text { Semi-major axis and } \\
\text { semi-minor axis of the } \\
\text { ellipse respectively. }\end{array}$ & $p, q$ & $\begin{array}{l}\text { Center of } \\
\text { the ellipse }\end{array}$ \\
\hline
\end{tabular}

Fig. 24. Definitions of node and edge attributes in attribute relational graph where nodes correspond to geometrical shapes. 


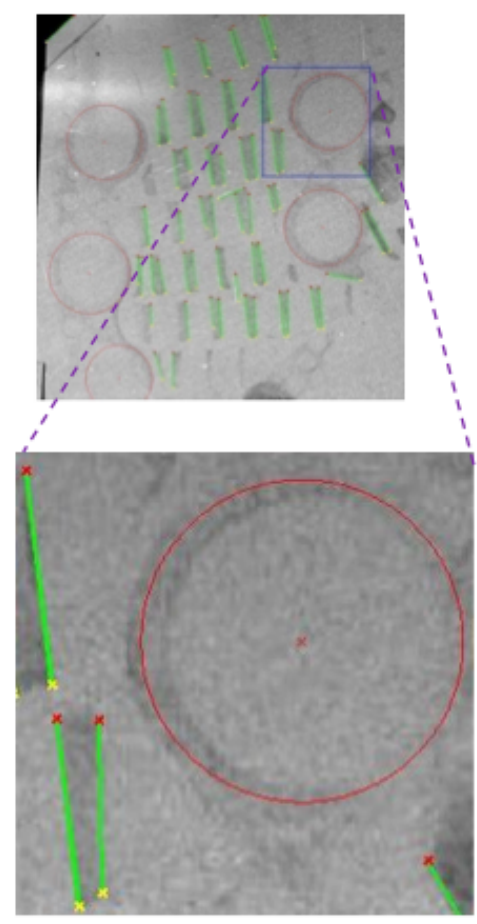

(a)

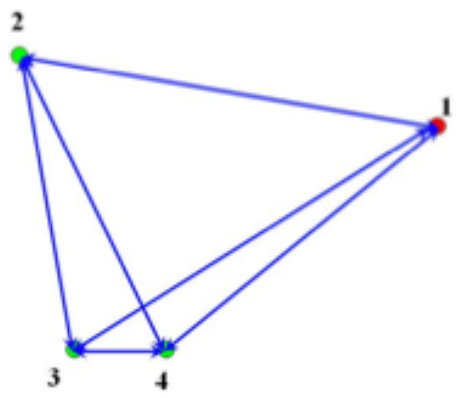

(c)

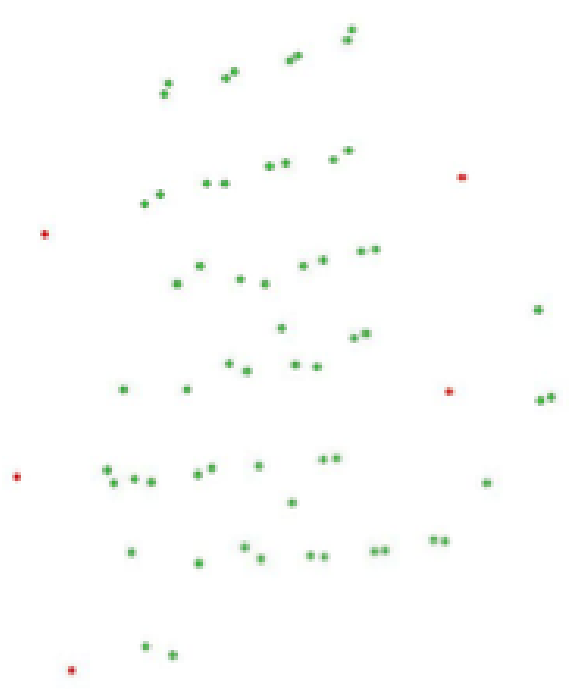

(b)

\begin{tabular}{|c|l|}
\hline Nodes and Edģes & \multicolumn{1}{|c|}{ Attributes } \\
\hline Node 1 & 0.61360 .8119 \\
\hline Node 2 & 0.0556 \\
\hline Node 3 & 0.0556 \\
\hline Node 4 & 0.0859 \\
\hline E12 & 0.47390 .7314 \\
\hline E13 & 0.52630 .7314 \\
\hline E14 & 0.56820 .7114 \\
\hline E21 & 0.52610 .73140 .1605 \\
\hline E23 & 0.00000 .54170 .6053 \\
\hline$\cdots$ & \\
\hline$\cdots$ & \\
\hline$\cdots$ & \\
\hline
\end{tabular}

(d)

Fig. 25. Attribute Relational Graph: (a) circles and straight lines in scene image with magnification of a portion showing three straight lines and a circle, (b) centers of all straight lines and circles, (c) graph for the two straight lines and circle, and (d) attributes of nodes and edges.

$$
\sum_{i=1}^{n_{1}} f_{i j} \leq w_{2 j}, \forall 1 \leq j \leq n_{2}
$$




$$
\sum_{i=1}^{n_{1}} \sum_{j=1}^{n_{2}} f_{i j}=\min \left(\sum_{i=1}^{n_{1}} w_{1 i}, \sum_{j=1}^{n_{2}} w_{2 j}\right) \text {. }
$$

Constraint 8 allows moving "supplies" from $P_{1}$ to $P_{2}$ and not vice versa. Constraint 9 limits the amount of "supplies" that can be sent by the bins in $P_{1}$ to their weights. Constraint 10 limits the bins in $P_{2}$ to receive no more "supplies" than their weights. Constraint 11 forces to move the maximum amount of "supplies" possible. This amount is referred to as the total flow in the transportation problem.

This is a linear programming problem which is solved efficiently by the transportation simplex algorithm [54]. Once the flow matrix F is found, the Earth Mover's Distance is defined as the overall work normalized by the total flow

$$
\operatorname{EMD}\left(P_{1}, P_{2}\right)=\frac{\sum_{i=1}^{n_{1}} \sum_{j=1}^{n_{2}} c_{i j} f_{i j}}{\sum_{i=1}^{n_{1}} \sum_{j=1}^{n_{2}} f_{i j}} .
$$

The computation of EMD assumes that there exists a proper distance measure to compute ground distance matrix $\mathbf{C}$, where the element $c_{i j}$ is the unit distance between a pair of bins $P_{1 i}$ and $P_{2 j}$, i.e. the work required to move one unit of "supplies" from the source bin $P_{1 i}$ to the destination bin $P_{2 j}$. It is straightforward to define such a distance between histogram bins because of their strict relative order.

Modification of EMD for Footwear Outsole Patterns. Robust ARG matching requires an assignment algorithm that yields not only a correspondence between two sets of vertices but also the similarity between them. In EMD, the bins are replaced by vertices and relations between them. Both vertices (nodes) and relations (edges) have attributes associated with them. The vertices also have associated weights with them, which are useful in performing assignment. However, when matching two ARGs, the ground distance between two vertices depends not only on the two vertices themselves, but also is related to their incident edges. Therefore, computing the ground distance between two vertices, involves a combinatorial optimization procedure to establish correspondence as consistently as possible between the attributed trees rooted at vertices. Hence, direct application of the basic EMD algorithm cannot solve the ARG matching problem and it needs to be augmented with a method for computing the ground distance matrix between all pairs of nodes.

Nested structure of EMD has been used to achieve robust ARG matching in computer vision [55]. However,it does not work well when two graphs to be matched have multiple attributes of different scales, and the difference in each attribute between two ARGs contribute unequally to the resulting overall distance. In this case, we need to apply appropriate weights on different attributes to balance their contributions to the overall distance, so that the difference in one feature/attribute will not dominate the overall distance. This step is essential as crime scene marks are created in an uncontrolled environment and they are highly degraded and partial, too. The weights for different attributes can be learnt using sensitivity analysis. First, we elaborate how learned weights are incorporated into EMD, followed by how to learn the weight vector.

A completely connected ARG is formally defined as $P=(V, R, n)$ where $V=\left\{V_{i} \mid 1 \leq i \leq n\right\}$ is the set of nodes and $R=\left\{R_{i j} \mid 1 \leq i, j \leq n\right\}$ is the set of relations between nodes. Each node has a weight and an attribute vector, $V_{i}=\left(w_{i}, \mathbf{v}_{i}\right)$ and each relation $R_{i j}$ has an attribute vector $\mathbf{r}_{i j}$. Let ARG of $1^{\text {st }}$ and $2^{\text {nd }}$ footwear prints be $F P_{1}=\left(V_{1}, R_{1}, n_{1}\right)$ and $F P_{2}=\left(V_{2}, R_{2}, n_{2}\right)$ respectively. To compute the Footwear print distance (FPD) between $F P_{1}$ and $F P_{2}$, an appropriate mapping $M$ between the two sets of nodes is needed. The cost or ground distance matrix is $\mathbf{C}=\left[c_{i j}\right]$ where $c_{i j}=c\left(V_{1 i}, V_{2 j} \mid V_{1 i} \in V_{1}, V_{2 j} \in V_{2}\right)$. The unit cost or distance between $V_{1 i}$ and $V_{2 j}$ is evaluated based on the similarity of the spatial configurations at the two nodes, which is explained later in this section.

By providing identical weights for all nodes the nested structure of EMD can handle the case of subgraph matching, i.e.,

$$
w_{1 i}=w_{2 j}=\frac{1}{\max \left(n_{1}, n_{2}\right)}, 1 \leq i \leq n_{1}, 1 \leq j \leq n_{2} .
$$

Unlike EMD, a node of $F P_{1}$ can transfer its weight to only one node of $F P_{2}$. This is known as uniqueness constraint. To enforce one-to-one correspondence, each node $i$ in the first ARG can match only one node $j$ in the second ARG or left unmatched, i.e. $f_{i j}$ may take the value of either $\frac{1}{\max \left(n_{1}, n_{2}\right)}$ or $0, \forall i \in\left\{1, \ldots, n_{1}\right\}, j \in$ $\left\{1, \ldots, n_{2}\right\}$. Therefore, we rewrite Eq. 12 as 


$$
\operatorname{FPD}\left(F P_{1}, F P_{2}\right)=\frac{\frac{1}{\max \left(n_{1}, n_{2}\right)} \sum_{\left\{(i, j) \mid f_{i j}>0\right\}} c_{i j}}{\sum_{\left\{(i, j) \mid f_{i j}>0\right\}} f_{i j}}
$$

The total number of correspondence pairs between the two ARGs is min $\left(n_{1}, n_{2}\right)$ so the total amount of flow transferred from $F P_{1}$ to $F P_{2}$ is $\frac{\min \left(n_{1}, n_{2}\right)}{\max \left(n_{1}, n_{2}\right)}$. Substituting this term for the denominator in Eq. 14 we get,

$$
\operatorname{FPD}\left(F P_{1}, F P_{2}\right)=\frac{\sum_{\left\{(i, j) \mid f_{i j}>0\right\}} c_{i j}}{\min \left(n_{1}, n_{2}\right)}
$$

Cost determination between two nodes. For a given pair of nodes in two graphs, say $V_{1 i}$ and $V_{2 j}$, how one node is different from the other depends not only on the nodes, but also on how they relate to their respective neighbors in terms of distance, orientation, position etc. This means that the distance $c_{i j}$ between the two nodes should be evaluated based on the distance between an attributed relational sub-graph rooted at $V_{1 i}$ and attributed relational sub-graph rooted at $V_{2 j}$. Each attributed relational sub-graph is an Attributed Tree (AT) [56]. ARG \& Attributed tree for two sample prints are shown in Fig. 26. This leads to a nested structure of $A R G$ matching, which consists of inner and outer steps. For the outer step, the unit cost or distance between $V_{1 i}$ and $V_{2 j}$, is defined as

$$
c\left(V_{1 i}, V_{2 j}\right)=\operatorname{EMD}\left(A T_{V_{1 i}}, A T_{V_{2 j}}\right),
$$

where $A T_{V_{1 i}}$ and $A T_{V_{2 j}}$ are attributed trees rooted at $V_{1 i}$ and $V_{2 j}$ in the two ARGs. The tree $A T_{V_{1 i}}$ consists of the root vertex $V_{1 i}$ and its connection to the rest of the $n_{1}-1$ vertices.

To calculate the distance between the two trees $A T_{V_{1 i}}$ and $A T_{V_{2 j}}$ using EMD framework, we build the inner cost matrix $\hat{\mathbf{C}}=\left[c_{\hat{i} \hat{j}}\right]$ whose elements correspond to pairwise node-to-node $\left(V_{1 \hat{i}}\right.$ to $\left.V_{2 \hat{j}}\right)$ distances in the two trees. The inner cost between $V_{1 \hat{i}}$ and $V_{2} \hat{j}$ takes into account not only the unary attributes of the nodes but also their edges attributes and is calculated by

$$
c\left(V_{1 \hat{i}}, V_{2 \hat{j}}\right)=\alpha d_{E}\left(\mathbf{v}_{1 \hat{i}}, \mathbf{v}_{2 \hat{j}}\right)+(1-\alpha) d_{E}\left(\mathbf{Q} * \mathbf{r}_{1 i \hat{i}}, \mathbf{Q} * \mathbf{r}_{2 j \hat{j}}\right)
$$

where $\alpha$ is a weight co-efficient in the interval $[0,1], d_{E}$ is the Euclidean distance, $\mathbf{r}_{1 i \hat{i}}$ is the attribute vector of the edge between $V_{1 i}$ and $V_{1 \hat{i}}$, Q is the weight vector and the operator '*' denotes the element-wise product between two vectors. Parameter $\alpha$ reflects the relative importance of the difference of node attributes and the difference of edge attributes in the evaluation of inner cost between two nodes, and is set to 0.5 assuming equal importance. Weight vector $\mathrm{Q}$ for all edge attributes is derived using sensitivity analysis.

Nodes $V_{1 i}$ and $V_{2 j}$ may have one of three possible labels: ' $\mathrm{L}$ ', 'C' and 'E' corresponding to lines, circles, or ellipses respectively. Thus there are 9 combinations of labels for $\left(V_{1 i}, V_{2 j}\right)$. A line cannot match with a circle or an ellipse regardless of their attributes and neighbors; while a circle and ellipse can match to some degree. Thus the unit matching cost for non-matching label pairs is $c\left({ }^{\prime} \mathrm{L}\right.$ ', ' $\mathrm{C}$ ') $=c\left({ }^{\prime} \mathrm{L}\right.$ ', ' $\mathrm{E}$ ') $=1$. For other label pairs, the node-to-node inner costs are determined using Eq. 17.

Computing the Weight Vector using Sensitivity Analysis. The distance between ARGs has different sensitivities for different attributes. The weight vector $\mathbf{Q}$ in Eq. 17 takes care of the differences in sensitivities. For large $n_{1}, \frac{2 n_{1}}{\left(2 n_{1}-1\right)} \approx 1$, thus we have $Q_{k} \approx \frac{1}{\sqrt{m}}$. When $n_{1}=2, Q_{k}=\frac{4}{3 \sqrt{m}}$. This indicates that we can determine the weights $\left\{Q_{k}, k=\{1, \ldots, m\}\right\}$ by first deriving the value of $Q_{k}$ in the case of 2-nodes, then multiplying it by $\frac{3}{4}$. The contribution of each edge attribute for all pairs of nodes to distance can be calculated as $\frac{\frac{2 n_{1}}{\left(2 n_{1}-1\right) * \sqrt{n}} * 1 * n_{1}\left(n_{1}-1\right) * \alpha}{n_{1}^{2}}=\frac{n_{1}-1}{\left(2 n_{1}-1\right) \sqrt{n}}$.

Examples. An example of distance computation with two simple prints and their graphs is shown in Fig. 26. Print $P_{1}$ has five imperfect elements: three circles, an ellipse and a straight line, its ARG has five nodes $\left\{V_{11}, . . V_{15}\right\}$. Print $P_{2}$ has six imperfect elements: two circles, one ellipse and three straight line segments, its ARG has six nodes $\left\{V_{21}, . . V_{26}\right\}$. Thus the number of edges in their ARGs are $2 \times\left(\begin{array}{l}5 \\ 2\end{array}\right)=20$ and $2 \times\left(\begin{array}{l}6 \\ 2\end{array}\right)=30$ respectively. 

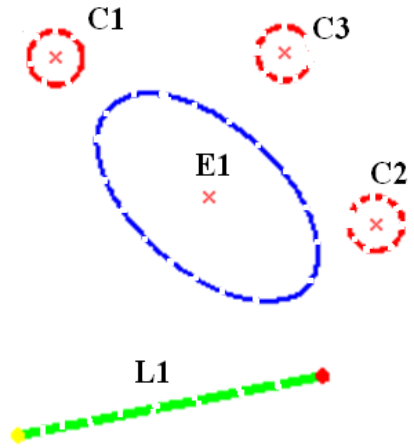

(a)

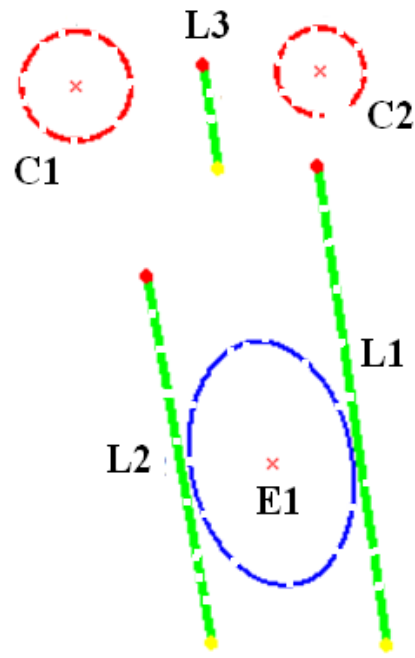

(d)

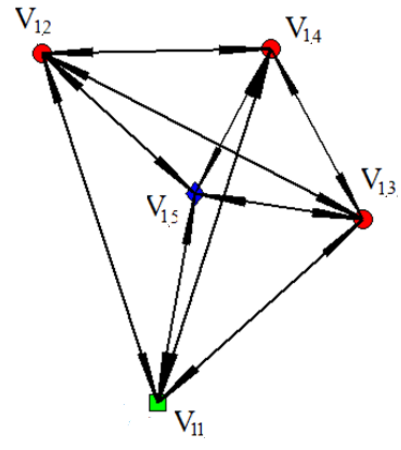

(b)

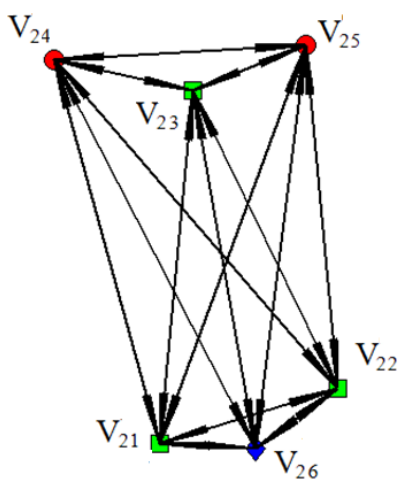

(e)

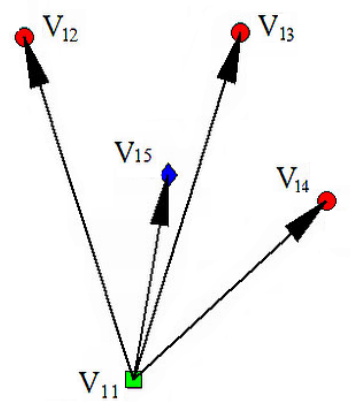

(c)

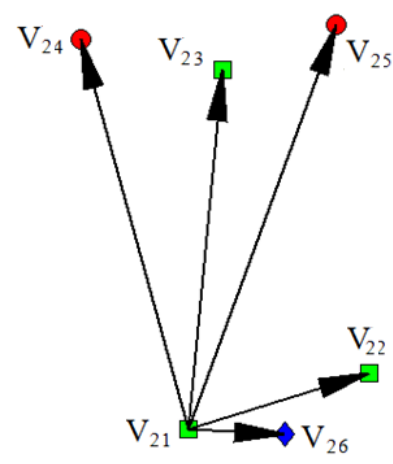

(f)

Fig. 26. Distance computation between two simple prints: (a) print $P_{1}$ with five primitive elements, (b) attributed relational graph of $P_{1}$ with vertices $V_{11} . . V_{15}$, (c) attributed tree rooted at $V_{11}$, (d) print $P_{2}$ with six elements, (e) attributed relational graph of $P_{2}$ with vertices $V_{21} . . V_{26}$ and (f) attributed tree rooted at $V_{21}$. Nodes represented by squares, circles and diamonds represent lines, circles and ellipses respectively. Using attributes of nodes and edges as defined in Figure 24 the distance evaluates to 0.5674 . 
The process of similarity computation in a more realistic scenario involving actual footwear prints is shown in Figure 27. In this case the distance evaluates to a much smaller value of 0.0835 indicating a finer degree of match.

Sensitivity analysis [57] is a system validation technique which can be used to determine robustness of the distance measure when the inputs are slightly disturbed. Its application here is to determine as to how sensitive the distance measure is to changes with respect to attributes. Plots of distance with respect to each of the attributes is obtained. A linear change is consistent with human perception whereas nonlinear behavior needs justification for its suitability. This analysis showed linear correlation with most attributes.

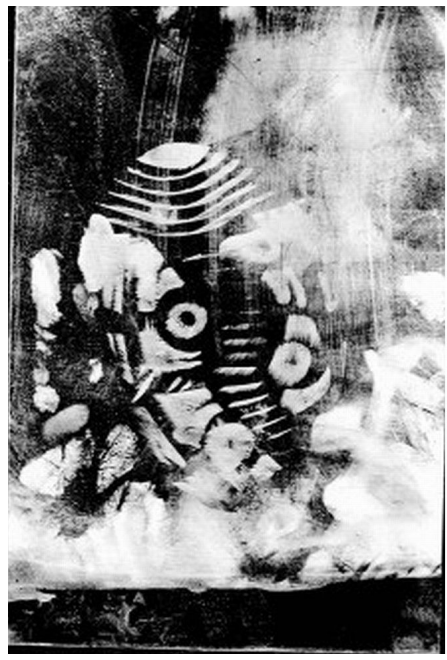

(a)
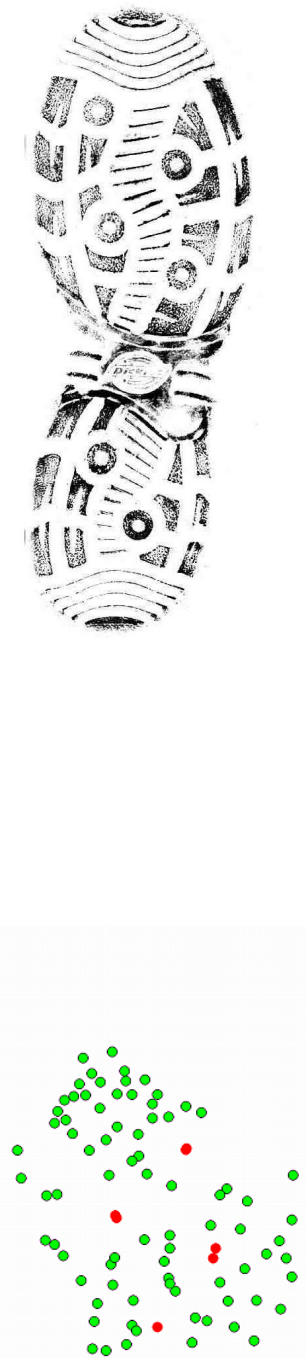

(c)
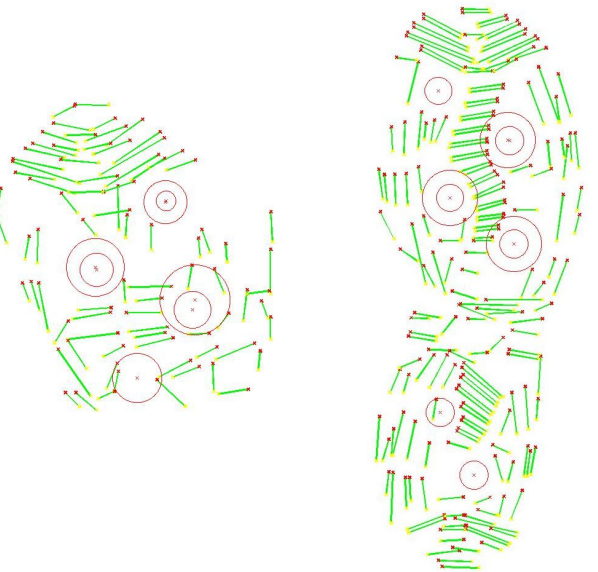

(b)

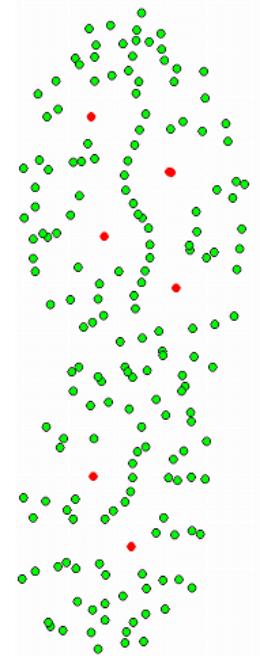

(d)

Fig. 27. Example of similarity computation between a crime scene image and a known outsole pattern: (a) input image and pattern, (b) geometric primitives detected in both, and (c,d) corresponding ARGs, where only nodes are shown for clarity. The distance between the two ARGs is 0.0835. 


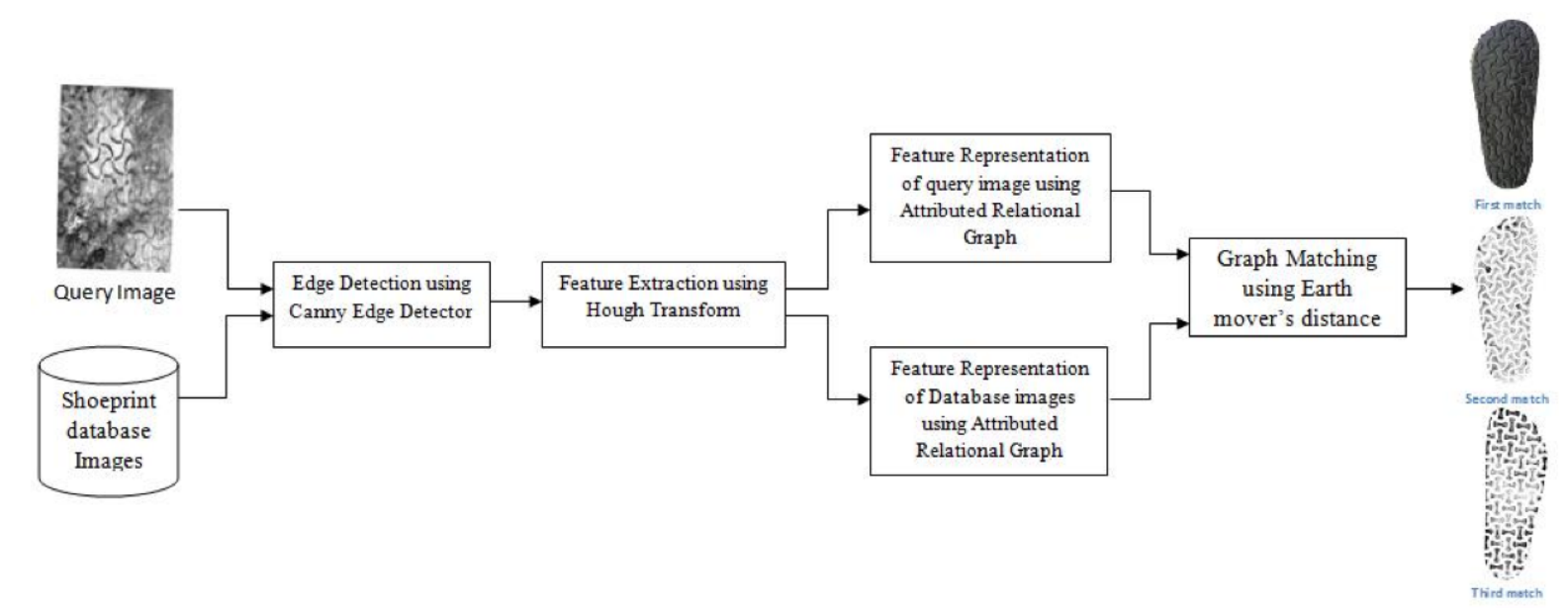

Fig. 28. Functional architecture for matching a query image with a database of sole-print reference patterns.

\subsection{Search Algorithms}

A functional block-diagram of an end-to-end system to retrieve closest matches to a query crime scene image in a database of reference images is shown in Figure 28. Image enhancement operations such as edge detection or contextually based image pixel labeling are performed on both the input and the known images. Next, a feature representation is constructed for the image either by extracting them from the entire image or by detecting local patterns in outsoles. The design should attempt to integrate several levels of analysis: (i) global shoe properties: heavily worn or brand new, shape, size etc., (ii) detailed and distinctive local features should be utilized to increase the discriminative power in order to confirm a match. Each level requires a different variety of image analysis techniques from robust geometric and texture feature detectors to detailed correlation of distinctive minutiae and their spatial arrangement.

A similarity measure appropriate to the feature description is used in the comparison of two images. In the design shown a graph representation of the characteristic features is used- where each node denotes a single geometrical primitive, such as a circle, an ellipse, a line segment, with attributes describing unary features of this primitive; each attributed edge between a pair of nodes represents spatial relationships between them. Thus the problem of image retrieval and matching is converted to an attributed graph matching problem. It involves establishing correspondence between the nodes of the two graphs. Retrieving the most similar prints to an impression can be made faster by clustering the database prints beforehand.

Reference Pattern Clustering. The computational complexity of distance computation for two ARGS with $n_{1}$ and $n_{2}$ nodes is $O\left(n_{1}^{2} n_{2}^{2} \max \left(n_{1}, n_{2}\right)\right)$. Since the computation is intensive, it is necessary to use approximate methods to speed-up the retrieval process. One approach is to eliminate several edge evaluations. Another is to cluster the reference images so that not all comparisons need to be made.

Clustering algorithms can be generally divided into partition-based, density-based and hierarchical based methods [58]. Algorithms like $k$-means, hierarchical clustering, and expectation maximization requires similarity matrix consisting of pair-wise distance between every footwear prints in dataset. Building similarity matrix is computationally expensive for a large dataset. Further, the ARG representing a footwear print has 200-300 nodes on average and nodes can vary considerably in terms of relative size, position etc. This makes the feature space very sparse and therefore similar footwear prints tend to stay close to each other and dissimilar ones stay apart. Hence, to cluster the entire dataset we use recurring patterns as fixed cluster centers [18].

Footwear outsoles typically contain recurring patterns such as waves and concentric circles [5, 59]. Each such pattern can represent a group of similar patterns. Each pattern is simple and its graph structure has a small number of nodes. Further, the ARG representing a footwear print has 200-300 nodes on average and nodes can vary considerably in terms of relative size, position etc. This makes the feature space very sparse 
and therefore similar footwear prints tend to stay close to each other and dissimilar ones stay apart. Hence, to cluster a huge dataset recurring patterns can be used as cluster representatives, which serve as initial seed clusters [60]. From visual inspection of 2,660 prints, 33 recurring patterns were determined and used as cluster representatives (see Figure 29). For each reference image, its distance to each pattern is computed and then assigned it to the nearest cluster representative. These cluster representatives are similar to cluster means in $k$-means algorithm but these "means" are fixed. Efficiency is achieved by exploiting sparseness of the feature space.
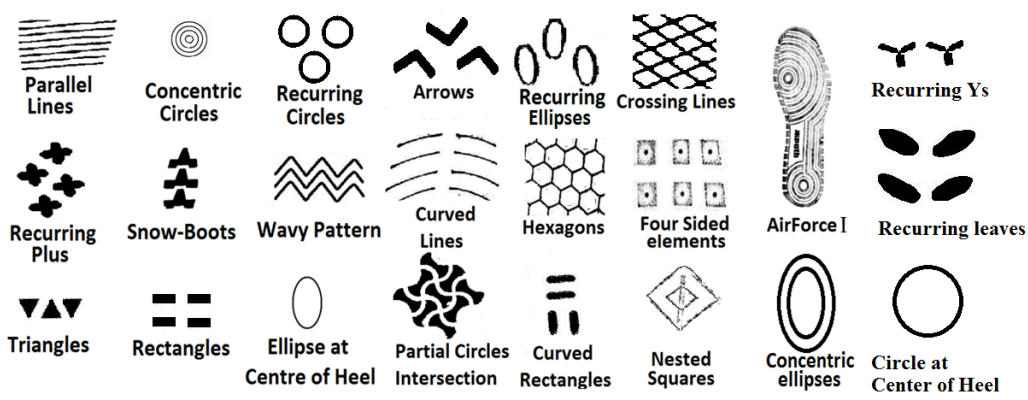

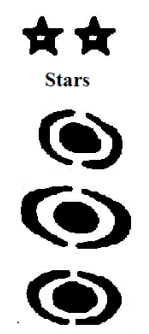

Ellipse from toe to center of the print

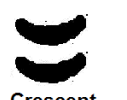

Crescent 负

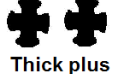

S Thick Ys Pentagons
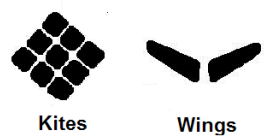

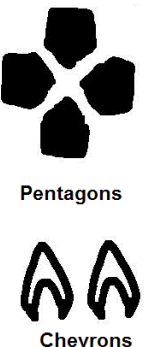

Chevrons

Fig. 29. Recurring patterns in outsole prints that are used as canonical cluster centers.

Clustering Step 1. The first step in feature extraction is to perform morphological operations such as dilation and erosion (Figure 30). This makes the interior region of the boundary uniform and hence the edge detector [61] does not detect any edges inside the boundary. This helps to enhance the quality of the edge image.

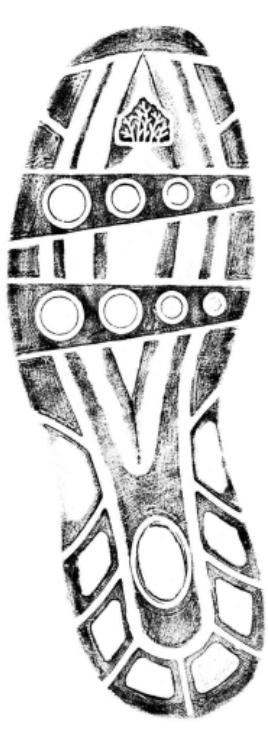

(a)

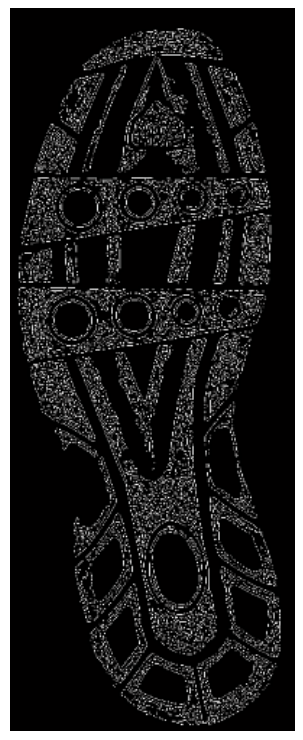

(b)

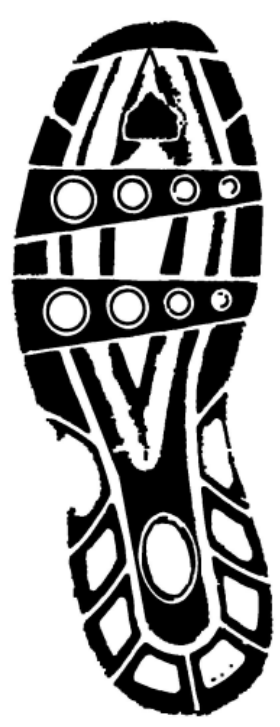

(c)

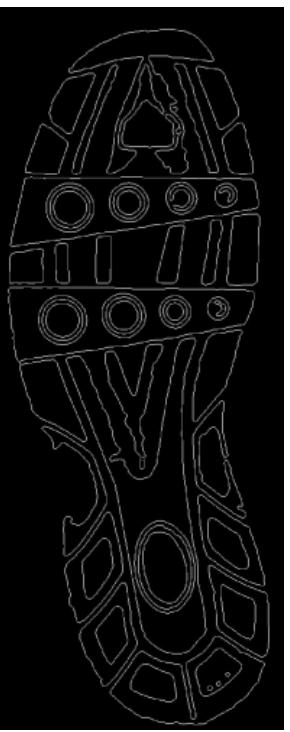

(d)

Fig. 30. Illustration of step 1 of clustering, where morphological operations are performed on reference patterns: (a) an input grey-scale image, (b) edge image of (a), (c) result of morphological operation on (a), (d ) edge image of (c). 
Clustering Step 2. The simple Hough transform (SHT) is used to detect circles in footwear prints. Pixels of detected circles are removed from the edge image and fed as input for ellipse detection using the randomized Hough transform (RHT). Pixels of detected ellipses are removed from the edge image and the output is fed as input for line detection. Features are extracted in the order: circle, ellipse and line (Figure 31). This is because circles are degenerated ellipses and arbitrary shapes in footwear print are approximated by piecewise lines.

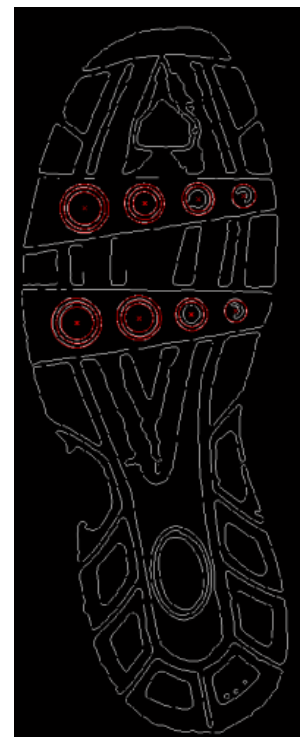

(a)

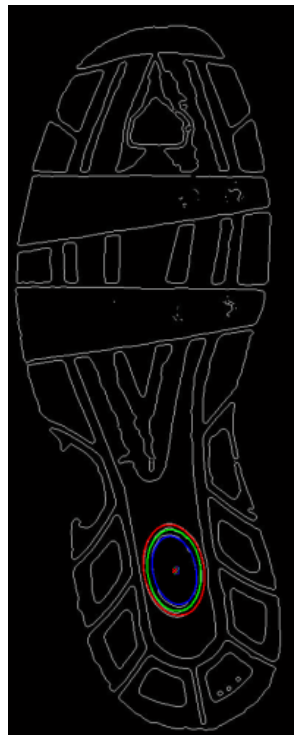

(b)

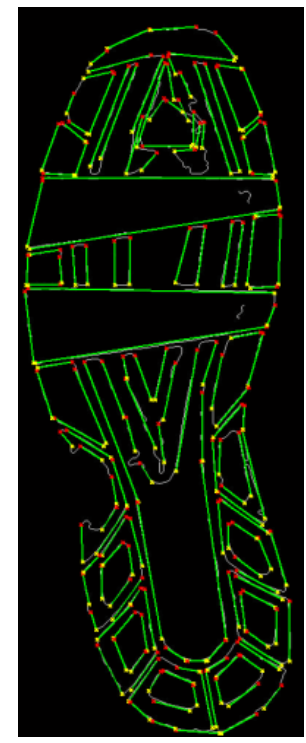

(c)

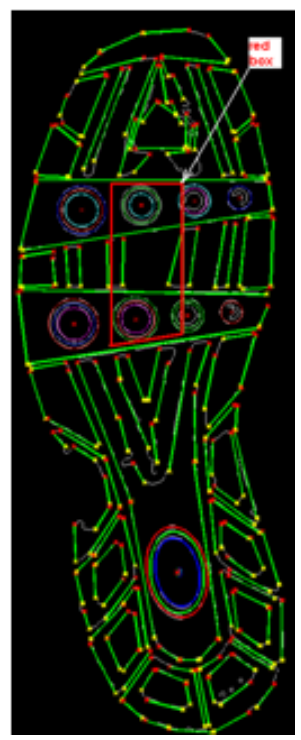

(d)

Fig. 31. Illustration of step 2 of clustering where the Hough transform is used to extract features. The sequence of operations is circle $\rightarrow$ ellipse $\rightarrow$ line. Detected features are: (a) circles. (b) ellipses. (c) line segments. (d) all features. Red box indicates a small region in the footwear print.

Clustering Step 3. For each detected feature, node attributes of completeness and quality of circle, eccentricity of the ellipse etc. are computed. Further, edge attributes like relative distance and position between nodes are calculated and finally an ARG is constructed (Figure 32). The distance between each reference print and every cluster representative is calculated. Then each print is assigned to the nearest representative, for which the distance is below threshold $T$. If distance between a print and cluster representatives are greater than $T$, then the print remains as a single cluster.

Clustering performance. With $T=0.15$, and 1,000 outsole patterns, the clustering algorithm assigned 550 patterns to one of 20 clusters whereas the remaining 450 were unique enough to be singleton clusters. Sample clusters based on the canonical patterns of Fig. 29 are shown in Fig. 33. Clustering accuracy is measured by the $F$-measure of retrieval, which is the weighted harmonic mean of precision and recall (Figure 34(a)). An advantage of using cluster centers is significant reduction in computation. For a database of 1000 prints, there are 499, 500 pairwise distances. With clustering based on $k$ recurring patterns as seed, $1000 \times k$ distance computations are needed; with $k=20$, computation is reduced by $96 \%$. This efficiency is achieved without compromising the accuracy or recall rate.

Retrieval performance. Evaluation metrics for retrieval performance are the cumulative match characteristic $(C M C)$ and speed. The CMC answers the question [11] "what is the probability of finding a match 


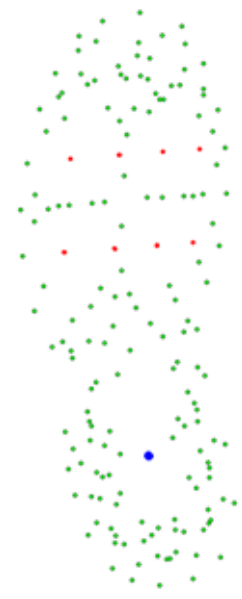

(a)

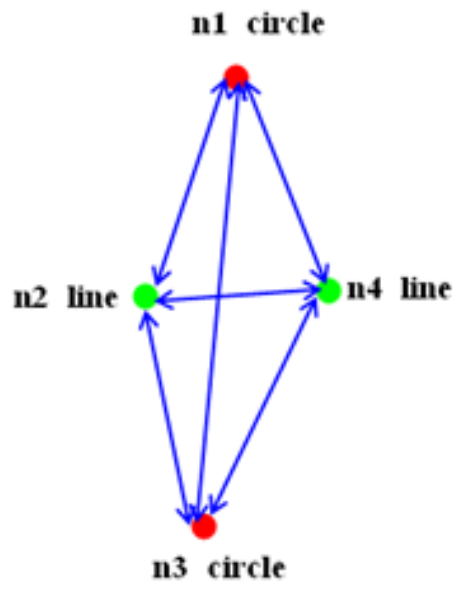

(b)

Fig. 32. Illustration of step 3 of clustering where a graph is constructed: (a) nodes in ARG of footwear pattern in Figure 30(a) with edges omitted due to complete connectivity, (b) subgraph for region enclosed within red box of Figure 31(d).

in the first $n$ percent of database images?" The cumulative match score is the proportion of times when the correct reference print is in the first $n$ percent of the sorted database. This metric can be used even when there is a single match in the database. For a dataset of 50 crime scene prints, used as query, and 1066 reference patterns, containing meta data such as brand and model, the CMC curve before clustering is shown in Fig.34(b). The CMC curve after clustering, where the query is matched against the cluster representatives to find the closest cluster and then against each pattern in the cluster to retrieve the top $n$ matches, does not show significant degradation. From the CMC curve, the top $0.1 \%$ of database patterns will contain the correct match with probability of 0.43 . Tests with crime scene marks have an error of $0.08 \%$ - the confidence interval for the sample size is the interval [0.03\%, 0.18\%].

The CMC of ARG-EMD is much better than that of the SIFT feature descriptor [43] also shown in Figure 34(b). SIFT, which is commonly used in image retrieval including Google's similar image search, performs only slightly better than randomly selecting a reference pattern. While SIFT features are not preserved among different outsoles of the same class and through wear lifetime, ARG-EMD extracts durable geometric features (of lines, ellipses, and their relationships) and demonstrates invariance to scale and rotation. ARGEMD has additional desirable properties: allows partial matching in a natural way, is robust to the change of the relational structure, and consistent with perceptual similarity (as can be seen in the two examples of Figure 35).

Speed. The time for processing a scene and reference image depends on the number of nodes in each graph. If the average time to compute one distance is 30 seconds then for a single query and 1,000 database entries, it takes 20-30 minutes. In a large reference database, the efficiency(speed) of retrieving a query print becomes important. Effective indexing techniques should be designed to enter standard reference patterns. Speed can be improved by: (i) reducing the number of nodes by merging two detected lines which are associated with a single straight boundary (to be done), (ii) using pre-filtering to enhance the speed performance e.g., computing the Euclidean distance between global feature vectors of the query print and each database print, and ignoring those database prints too far from the query to be a potential match, (iii) relaxing full connectivity in graph by triangulation, and (iv) other improvements. In terms of performance, with 50 scene images the average time was 120 minutes before clustering and 8 minutes after with no significant retrieval degradation. 


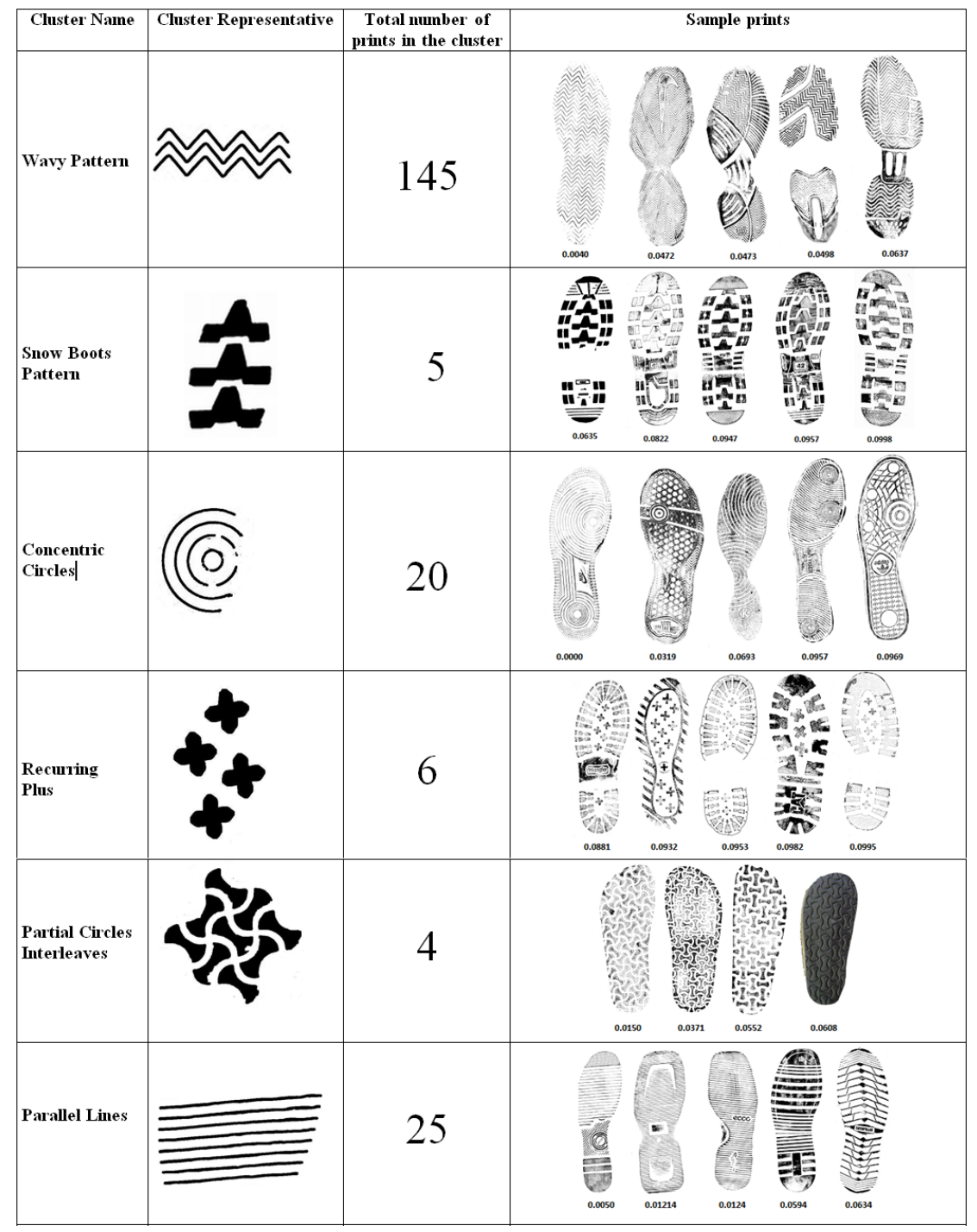

Fig. 33. Sample clusters of reference patterns based on using the canonical patterns in Fig. 29.

\subsection{Quantifying uncertainty of match}

In reporting the results of a comparison between the evidence and known an expression of the uncertainty involved is useful. This opinion can be expressed in probabilistic terms using statistical methods for computing the strength of evidence [62]. A rule for converting likelihood ratios into scales has also been suggested[63].

For evidence interpretation, three different approaches have been stated: "Classical", "Likelihood Ratio" and "Full Bayes' Rule". The likelihood ratio approach [64] is widely accepted among various forensic investigations as it provides a transparent, consistent and logical framework to discriminate among competing hypotheses. In the Full Bayes' Rule approach, the posterior probability of a set of hypotheses given the existing evidence is determined. Although this method has been a very common practice of forensic document examiners in central European countries, it has been said that there is no creditable justifications for its validity and appropriateness[65].

The likelihood ratio is the ratio of the two probabilities of the evidence given two competing hypotheses: $h^{0}$ - the crime scene print is created by the same footwear as the known print and $h^{1}$ - the crime scene print is not from the known. This ratio can be expressed as: $L R=\frac{P\left(E \mid h^{0}, I\right)}{P\left(E \mid h^{1}, I\right)}$. where $E$ is the evidence given 


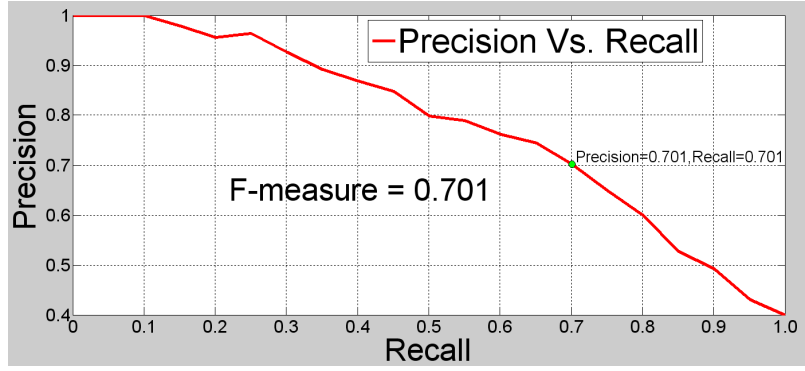

(a)

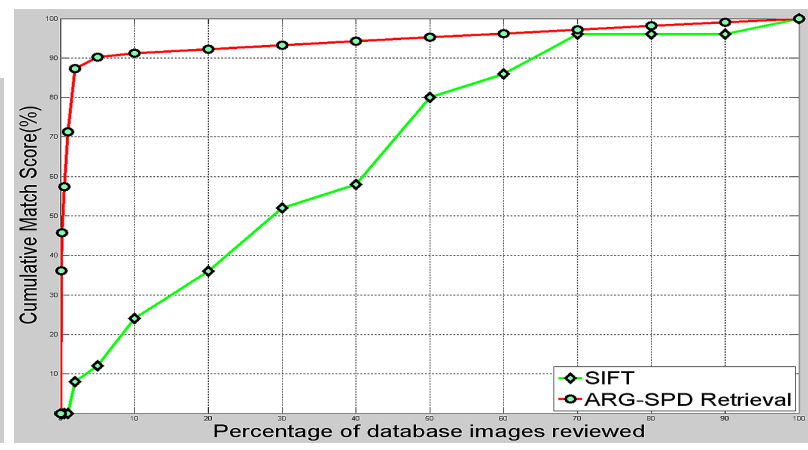

(b)

Fig. 34. Retrieval performance: (a) precision-recall curve for circles only, and (b) cumulative match characteristic, which gives the probability of correct match in the top $n \%$ of ranked database, of ARG-EMD (based on circles, ellipses and straight lines) compared with that of SIFT.

by the crime scene mark, and $I$ is all the background information relevant to this case. This approach can be decomposed into the following three steps: (i) estimate the within-class and between-class shoe-print variability, (ii) compute the LR for the evidence, and (iii) convert the LR into a verbal scale.

Degradation Model. In order to obtain a probabilistic measure it is necessary to characterize within-class and between-class variabilities. Within-class variability measures the variance of features of multiple prints from the same outsole. To be able to simulate different variations caused by wears, incompleteness and the change of medium and illumination, we can apply image degradation models multiple times on each database image to produce a set of degraded outsole prints.

Approximating the Likelihood Ratio. Direct computation of the likelihood ratio is infeasible due to the large number of possible variations of the same and different distributions. Since uncertainty is a function of similarity of the characteristics (both class and individualizing) as well as the rarity of the characteristics [66] the following methods based on (i) distance and (ii) distance and rarity can be used.

Distance Method. A matching algorithm can be applied to calculate the distance between each pair of within-class prints. It is then possible to build a probability distribution of within-class distance. Betweenclass variability measures the variance of features of multiple prints that are from different classes. In a similar way the within-class variability can be modeled. Given a distance between the crime scene mark and a test mark made by the suspect's shoe, we can compute the likelihood of the observed distance $d$ given the hypothesis that the two marks are from the same source, as well as the likelihood of the distance given the hypothesis that the two marks are from different sources. The ratio of these two likelihoods is then calculated to get $L R_{D}=\frac{P\left(d \mid h^{0}\right)}{P\left(d \mid h^{1}\right)}$.

Using footwear impressions together with ground truth, histograms for $P\left(d \mid h^{i}\right)(i=0,1)$ are built as shown in Fig. 36; in this example 1,060 degraded footwear prints with ground truth were used. Modeling $P\left(d \mid h^{0}\right)$ by a Gaussian $\mathcal{N}\left(d \mid \mu_{0}, \sigma_{0}^{2}\right)$, and $P\left(d \mid h^{1}\right)$ by a mixture of Gaussians the likelihood ratio is computed. The distribution of LRs, determined from a learning set, can be used to convert the LR value into an opinion scale.

Distance and Rarity Method. The distance method provides a severe approximation to the true likelihood ratio by going from a high-dimensional feature space to a one-dimensional distance space. A better approach, 

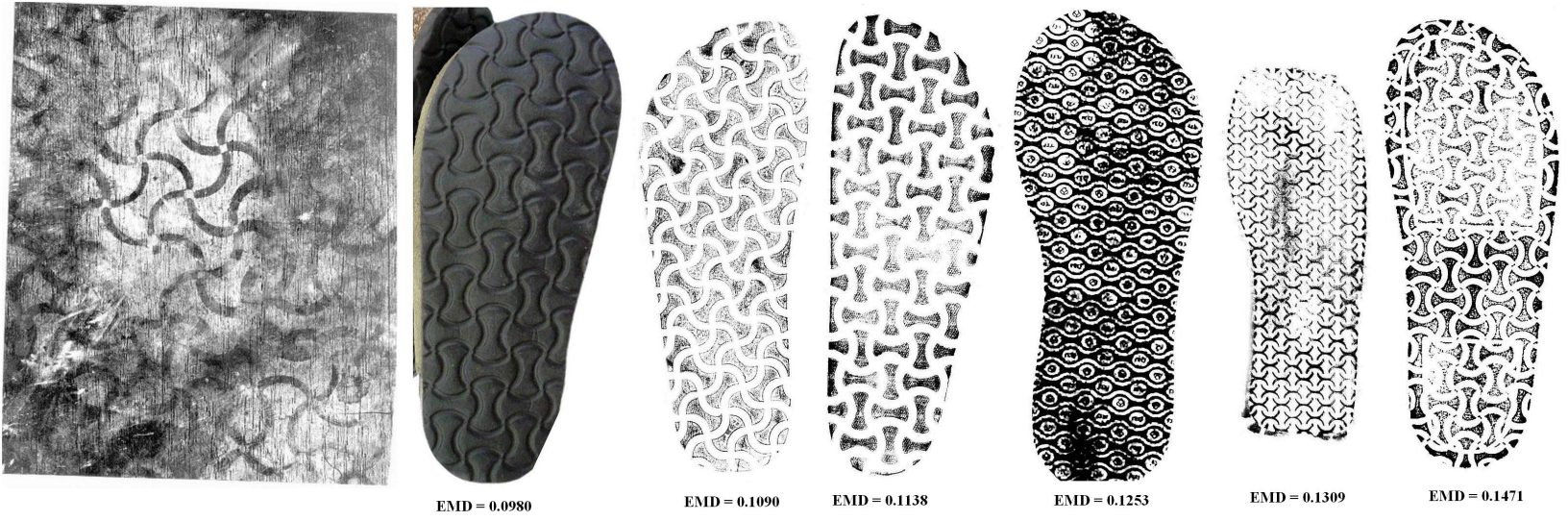

(a)
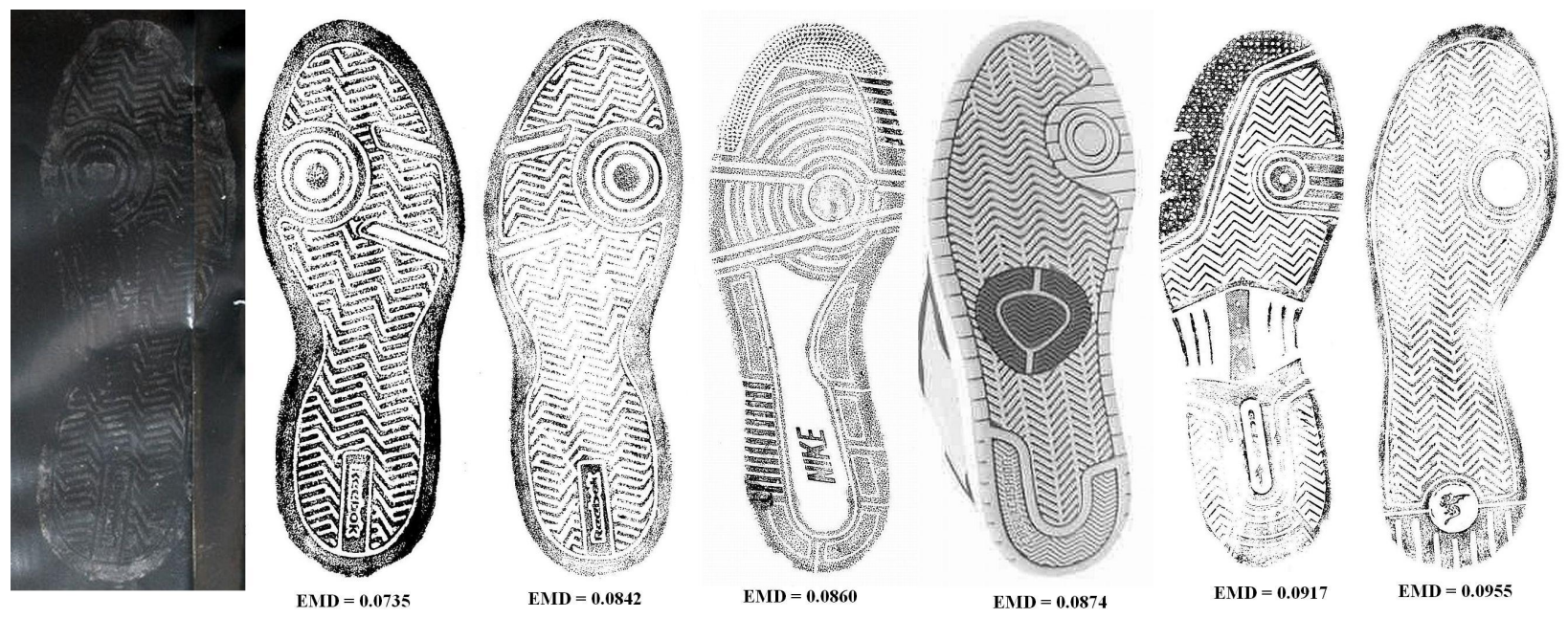

(b)

Fig. 35. Results of automatic retrieval with two queries shown as the left-most images followed on the right by the top database entries retrieved. It can be seen that the top choices are similar to human perception. 


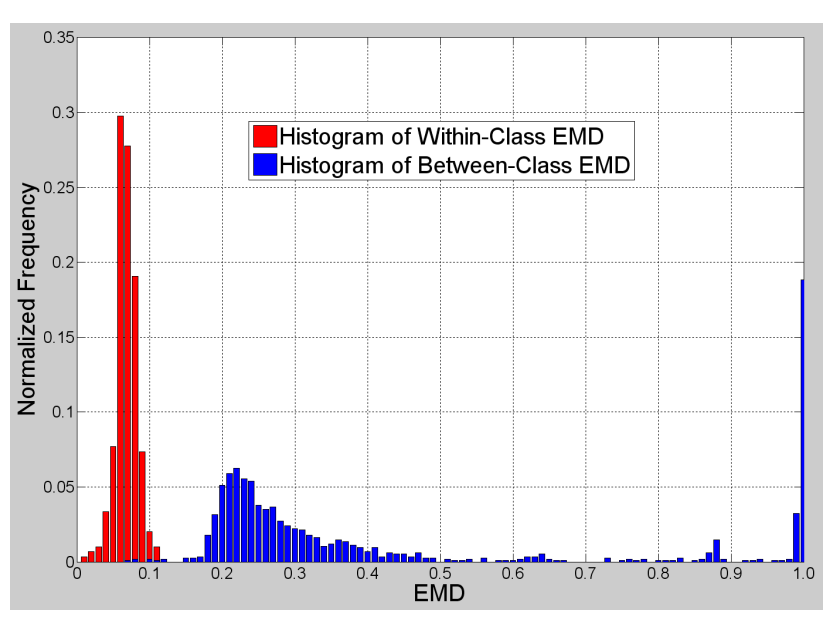

(a)

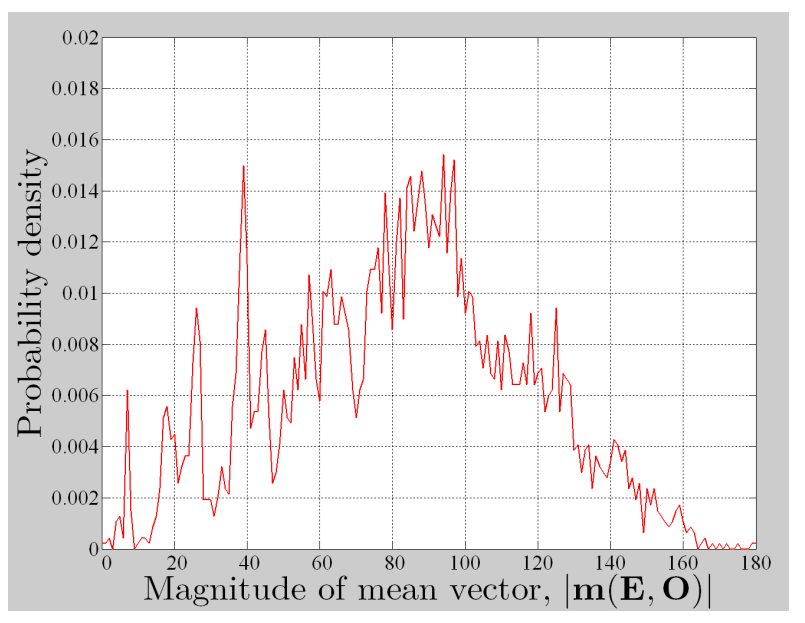

(b)

Fig. 36. Likelihood Ratio methods: (a) distance method is based on histograms of intra- and inter-class distance, and (b) distance and rarity method uses distribution of magnitude of mean vector $\mathbf{m}(\mathbf{E}, \mathbf{O}$ ) (built with 5,289 pairs of samples).

as shown in [66], is to estimate $L R$ as the product of two factors, one based on difference and the other on rarity:

$$
L R_{D R}=P\left(\mathbf{d}(\mathbf{o}, \mathbf{e}) \mid h^{0}\right) * \frac{1}{P(\mathbf{m}(\mathbf{o}, \mathbf{e}))},
$$

where $\mathbf{d}(\mathbf{o}, \mathbf{e})$ is the difference between object vector $\mathbf{o}$ and evidence vector $\mathbf{e}$, and $\mathbf{m}(\mathbf{o}, \mathbf{e})$ is the mean of $\mathbf{o}$ and $\mathbf{e}$.

When features are extended from vectors to graphs associated with feature sets $\mathbf{E}$ and $\mathbf{O}$, correspondence between them is not apparent, and the number of corresponding elements (features) in $\mathbf{E}$ and $\mathbf{O}$ may be different. Instead of defining a distribution on the set difference $\mathbf{E}-\mathbf{O}$ we can use the distribution of the distance $d(\mathbf{E}, \mathbf{O})$. Next we discuss an approximation to the rarity term.

In computing the distance between two ARGs, we determine corresponding nodes between them, This induces two sub-graphs in the two original ARGs that have an equal number of nodes and edges. We construct a feature vector from each of the two sub-graphs. The scalar distance (if computed by Euclidean distance) is the magnitude of the vector difference. By analogy, we can use the distribution of the magnitude of the mean vector as a substitute for the distribution of the mean vector itself, i.e.

$$
L R_{D R}=\frac{P\left(\mathbf{d}(\mathbf{E}, \mathbf{O}) \mid h^{0}\right)}{P(\mathbf{m}(\mathbf{E}, \mathbf{O}))} \approx \frac{P\left(d(\mathbf{E}, \mathbf{O}) \mid h^{0}\right)}{P(|\mathbf{m}(\mathbf{E}, \mathbf{O})|)} .
$$

This approximation has intuitive appeal: two graphs with more matched features will have a greater value of $|\mathbf{m}(\mathbf{E}, \mathbf{O})|$ than with fewer matched features. By mapping the distribution of mean vector $\mathbf{m}(\mathbf{E}, \mathbf{O})$ of varied length to the distribution of its magnitude, we have overcome the difficulty of defining the distribution of the difference $\mathbf{E}-\mathbf{O}$, avoided normalization and made both numerator and denominator have the same dimension. This mapping does give a reasonable approximation of the original rarity. In experiments the FPD was computed between all pairs of prints in the training data set (1,060 prints) yielding an average error rate of $4.5 \%$ with the distance method and $2.5 \%$ with the distance and rarity method.

\section{Summary and Conclusions}

While footwear impressions are commonly found in crime scenes, they are not often used in either the investigative or prosecutorial phases of criminal justice due to many practical difficulties. Reliable automated 
tools should enable more use of footwear impression evidence. A review of methods of footwear print examination reveals the need for computational solutions for several tasks: enhancing the quality of crime scene images, representing outsole patterns so as to be useful in comparison, evaluating similarity between evidence and known, implementation of algorithms to retrieve closest matches in a reference database, performance evaluation metrics and quantifying uncertainty of opinion. Data sets useful in developing methods are: (i) simulated prints (crime scene prints obtained by stepping on talcum powder and then on carpet, and known prints by stepping on chemically treated paper), (ii) photographs of outsoles retrieved by a web crawler from shoe-vendor websites, and (iii) actual crime scene prints and corresponding known prints. Since results with simulated images tend to be over-optimistic results should focus on real crime scene prints.

For extracting foreground pixels from crime scene images, a method based on utilizing statistical dependencies between nearby pixels (one based on CRFs) is better than thresholding methods. For representing the geometrical patterns commonly found in outsole prints, a structural method performs better than simple two-dimensional (GSC) and three-dimensional (SIFT) representations. The structural method is based on detecting component geometric shapes, principally ellipses of different eccentricities. The relationships between these elements in the print is then modeled as a graph whose nodes represent primitive elements (together with defining attributes relating to parameters such as radius as well as quality in the image) and whose edges represent spatial relationships (also attributed with a list of characteristics). Given two patterns represented as graphs, their similarity is determined by using a graph distance measure, one related to measuring histogram distance and the Wasserstein metric. It characterizes similarity by a number ranging from 0 to 1 .

The retrieval task is to find the closest match to a crime scene print in a local/national database so as to determine footwear brand and model. This process is made faster if database prints are grouped into clusters of similar patterns. For this an ARG is constructed for each known print, where each node is a primitive feature and each edge represents a spatial relationship between nodes. The distance between ARGs is used as similarity measure. This distance is computed between each known print and a pre-determined set of canonical patterns to form clusters. By clustering known images into cognitively similar patters, higher efficiency is achieved in retrieval. The following topics of further research can be identified:

1. Statistical machine learning approaches can be used effectively in several phases such as enhancement of the crime scene image similarity computation, and drawing a conclusion,

2. A standardized database of crime scene marks would allow researchers to develop and benchmark the performance of their algorithms and systems.

3. Robustness and sensitivity of the similarity measures needs to be further studied, e.g., different sizes of the query image, increased number of footwear models, etc.

4. The use of the similarity metrics in the computation of likelihoods using both class characterizing and individualizing features need to be studied so as to provide uncertainty measures in comparison.

\section{References}

1. Bodziak, W.: Footwear Impression Evidence Detection, Recovery and Examination, second ed. CRC Press (2000)

2. Stone, R.S.: Footwear examinations: Mathematical probabilities of theoretical individual characteristics. Journal of Forensic Identification $\mathbf{5 6}$ (2006) 577-599

3. Geradts, Z., Keijzer, J.: The image-database REBEZO for shoeprints with developments on automatic classification of shoe outsole designs. Forensic Science International 82 (1996) 21-31

4. Alexander, A., Bouridane, A., Crookes, D.: Automatic classification and recognition of shoeprints. In: Proc. Seventh Internationl Conference Image Processing and Its Applications. Volume 2. (1999) 638-641

5. Girod, A.: Computerized classification of the shoeprints of burglar's shoes. Forensic Science International 1 (1982) 59-65

6. Bouridane, A., Alexander, A., Nibouche, M., Crookes, D.: Application of fractals to the detection and classification of shoeprints. In: Proceedings International Conference Image Processing. Volume 1. (2000) 474-477

7. Sawyer, N.: SHOE-FIT: A computerised shoe print database. In: Proc. European Convention on Security and Detection. (1995)

8. Ashley, W.: What shoe was that? the use of computerised image database to assist in identification. Forensic Science Int. 82(1) (1996) 7-20 
9. Foster, Freeman: Solemate. http://fosterfreeman.com (2010)

10. Bouridane, A.: Imaging for Forensics and Security: From Theory to Practice. First edn. Springer (2009)

11. deChazal, P., Flynn, J., Reilly, R.B.: Automated processing of shoeprint images based on the Fourier transform for use in forensic science. IEEE Trans. Pattern Anal. Mach. Intell 27 (2005) 341-350

12. Zhang, L., Allinson, N.: Automatic shoeprint retrieval system for use in forensic investigations. In: UK Workshop On Computational Intelligence. (2005)

13. Pavlou, M., Allinson, N.M.: Automatic extraction and classification of footwear patterns. In: Lecture Notes in Computer Science, Proc. Intelligent Data Engineering and Automated Learning. (2006) 721-728

14. Crookes, D., Bouridane, A., Su, H., Gueham, M.: Following the footsteps of others: Techniques for automatic shoeprint classification. Second NASA/ESA Conference on Adaptive Hardware and Systems (2007) 67-74

15. Gueham, M., Bouridane, A., Crookes, D.: Automatic classification of partial shoeprints using advanced correlation filters for use in forensic science. International Conference on Pattern Recognition (2008) 1-4

16. Patil, P.M., Kulkarni, J.V.: Rotation and intensity invariant shoeprint matching using gabor transform with application to forensic science. Pattern Recognition 42 (2009) 1308-1317

17. Dardi, F., Cervelli, F., Carrato, S.: A texture based shoe retrieval system for shoe marks of real crime scenes. Proc. International Conference on Image Analysis and Processing 5716 (2009) 384-393

18. Tang, Y., Srihari, S.N., Kasiviswanthan, H.: Similarity and clustering of footwear prints. In: IEEE Symposium on Foundations and Practice of Data Mining (GrC 2010), IEEE Computer Society Press (2010)

19. Tang, Y., Srihari, S.N.: Ellipse detection using sampling constraints. In: Proc. IEEE Int. Conf. Image Proc., IEEE Computer Society Press (2011)

20. Mikkonen, S., Astikainenn, T.: Database classification system for shoe sole patterns - identification of partial footwear impression found at a scene of crime. Journal of Forensic Science 39(5) (1994) 1227-1236

21. Huynh, C., de Chazal, P., McErlean, D., Reilly, R., Hannigan, T., Fleud, L.: Automatic classification of shoeprints for use in forensic science based on the Fourier transform. In: Proc. 2003 International Conference Image Processing. Volume 3. (2003) 569-572

22. Mikolajczyk, K., Schmid, C.: A performance evaluation of local descriptors. IEEE Trans. on Pattern Analysis and Machine Intel. 27 (2005) 1615-1630

23. Ghouti, L., Bouridane, A., Crookes, D.: Classification of shoeprint images using directional filter banks. International Conference on Visual Information Engineering (2006) 167-173

24. Su, H., Crookes, D., Bouridane, A.: Thresholding of noisy shoeprint images based on pixel context. Pattern Recognition Letters 28(2) (2007) 301-307

25. Sun, W., Taniar, D., Torabi, T.: Image mining: A case for clustering shoe prints. International Journal of Information Technology and Web Engineering 3 (2008) 70-84

26. AlGarni, G., Hamiane, M.: A novel technique for automatic shoeprint image retrieval. Forensic Science International 181 (2008) 10-14

27. Xiao, R., Shi, P.: Computerized matching of shoeprints based on sole pattern. Lecture Notes In Computer Science;Proceedings of the 2nd international workshop on Computational Forensics 5158 (2008) 96-104

28. Jingl, M.Q., Ho, W.J., Chen, L.H.: A novel method for shoeprints recognition and classification. International Conference on Machine Learning and Cybernetics 5 (2009) 2846-2851

29. Nibouche, O., Bouridane, A., Gueham, M., Laadjel, M.: Rotation invariant matching of partial shoeprints. International Machine Vision and Image Processing Conference (2009) 94-98

30. Cervelli, F., Dardi, F., Carrato, S.: Comparison of footwear retrieval systems for synthetic and real shoe marks. In: Proc. Sixth Intl. Symp. Image and Signal Processing and Analysis, Salzburg, Austria. (2009) 684-689

31. Dardi, F., Cervelli, F., Carrato, S.: A combined approach for footwear retrieval of crime scene shoe marks. Proc. ICDP-09, Third International Conference on Imaging for Crime Detection and Prevention, London, UK (2009) Paper No. P09

32. Wang, R., Hong, W., Yang, N.: The research on footprint recognition method based on wavelet and fuzzy neural network. International Conference on Hybrid Intelligent Systems (2009) 428-432

33. Otsu, N.: A threshold selection method from gray level histogram. IEEE Transaction on Systems, Man and Cybernetics 9 (1979) 62-66

34. Ramakrishnan, V., Srihari, S.N.: Extraction of shoeprint patterns from impression evidence using conditional random fields. In: Proceedings of International Conference on Pattern Recognition, Tampa, FL, IEEE Computer Society Press (2008)

35. Koller, D., Friedman, N.: Probabilistic Graphical Models. MIT Press (2009)

36. Shetty, S., Srinivasan, H., Beal, M., Srihari, S.: Segmentation and labeling of documents using conditional random fields. Document Recognition and Retrieval XIV SPIE Vol 6500 (2007) 65000U1-9

37. Kumar, S., Hebert, M.: Discriminative fields for modeling spatial dependencies in natural images. in Neural Information Processing Systems (NIPS) (2003) 
38. Wallach, H.: Efficient training of conditional random fields. Master's thesis, University of Edinburgh (2002)

39. Canny, J.: A computational approach to edge detection. IEEE Trans. Pattern Anal. Mach. Intell. 8(6) (1986) 679-698

40. Gonzalez, R.C., Woods, R.E., Eddins, S.L.: Digital Image Processing Using MATLAB $1^{\text {st }}$ ed. Prentice Hall (2003)

41. Rui, Y., Huang, S., Chang, S.: Image retrieval: Current techniques, promising directions, and open issues. Journal of Visual Communication and Image Representation 10 (1999) 39-62

42. Srihari, S.N., Huang, C., Srinivasan, H.: On the discriminability of the handwriting of twins. Journal of Forensic Sciences 53(2) (2008) 430-446

43. Lowe, D.: Distinctive image features from scale-invariant keypoints. International Journal of Computer Vision 60(2) (2004) 91-110

44. Hough, P.: Machine analysis of bubble chamber pictures. International Conference on High Energy Accelerators and Instrumentation, CERN (1959)

45. Srihari, S.N., Govindaraju, V.: Analysis of textual images using the Hough transform. Machine Vision and Applications 2 (1989) 141-153

46. Goulermas, J., Liatsis, P.: Incorporating gradient estimations in a circle-finding probabilistic hough transform. Pattern Analysis and Applications 26 (1999) 239-250

47. Wu, W.Y., Wang, M.J.J.: Elliptical object detection by using its geometric properties. Pattern Recognition 26 (1993) 1499-1509

48. McLaughlin, R.: Randomized Hough transform: better ellipse detection. IEEE TENCON-Digital Signal Processing Applications 1 (1996) 409-414

49. Haralick, R.M., Shapiro, L.G.: Computer and Robot Vision. Addison Wesley (1992)

50. Bunke, H., Irniger, C., Neuhaus, M.: Graph matching: Challenges and potential solutions. International Conference on Image Analysis and Processing 3617 (2008) 1-10

51. Sanfeliu, A., Fu, K.S.: A distance measure between attributed relational graphs for pattern recognition. IEEE transactions on systems, man, and cybernetics 13 (1983) 353-362

52. Bunke, H., Messmer, B.T.: Efficient attributed graph matching and its application to image analysis. In: Proceedings of the 8th International Conference on Image Analysis and Processing. (1995) 45-55

53. Rubner, Y., Tomasi, C., Guibas, L.J.: The earth mover's distance as a metric for image retrieval. International Journal of Computer Vision 40 (2000) 99-121

54. Hillier, F.S., Liebermann, G.J.: Introduction to Mathematical Programming. Second edition edn. McGraw-Hill (1995)

55. Kim, D.H., Yun, I.D., Lee, S.U.: Attributed relational graph matching algorithm based on nested assignment structure. Pattern Recognition 43 (2010) 914-928

56. Pelillo, M., Siddiqi, K., Zucker, S.: Many-to-many matching of attributed trees using association graphs and game dynamics. In: Proceedings of 4th international Workshop on Visual Form. (2001) 583-593

57. Smith, E., Szidarovszky, F., Karnavas, W., Bahill, A.: Sensitivity analysis, a powerful system validation technique. The Open Cybernetics and Systemics Journal 2 (2008) 39-56

58. Aldenderfer, M., Blashfield, R.: Cluster Analysis. SAGE (1984)

59. Mikkonen, S., Suominen, V., Heinonen, P.: Use of footwear impressions in crime scene investigations assisted by computerised footwear collection system. Forensic Science International 82(1) (1996) 67-79

60. Basu, S., Banerjee, A., Mooney, R.J.: Semi-supervised clustering by seeding. In: Proc. of the Nineteenth International Conference on Machine Learning. (2002) 27-34

61. Nixon, M., Aguado, A.: Pattern Extraction and Image Processing. Elsevier Science (2002)

62. Aitken, C., Taroni, F.: Statistics and the Evaluation of Evidence for Forensic Scientists. Wiley (2004)

63. Evett, I.: Towards a uniform framework for reporting opinions in forensic science casework. Science and Justice 38(3) (1998) 198-202

64. I. Evett, J., Lambert, Buckleton, J.: A Bayesian approach to interpreting footwear marks in forensic casework. Science and Justice 38(4) (1998) 241-247

65. Biedermann, A., Taroni, F.: Inadequacies of posterior probabilities for the assessment of scientific evidence. Law, Probability and Risk 4 (2005) 89-114

66. Tang, Y., Srihari, S.N.: Likelihood ratio estimation in forensic identification using similarity and rarity. Pattern Recognition 47(3) (2014) 945-958 